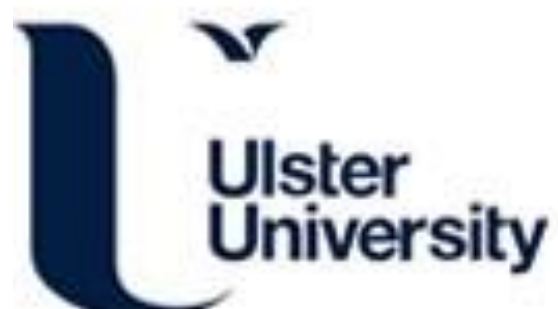

\section{Precision Printing of Customized Cylindrical Capsules with Multifunctional Layers for Oral Drug Delivery}

Li, X., Zhang, C., Wu, S., Chen, X., Mai, J., \& Chang, M. W. (2019). Precision Printing of Customized Cylindrical Capsules with Multifunctional Layers for Oral Drug Delivery. ACS Applied Materials and Interfaces, 11(42), 39179-39191. https://doi.org/10.1021/acsami.9b13568

Link to publication record in Ulster University Research Portal

Published in:

ACS Applied Materials and Interfaces

Publication Status:

Published (in print/issue): 23/10/2019

DOI:

10.1021/acsami.9b13568

Document Version

Author Accepted version

\section{General rights}

Copyright for the publications made accessible via Ulster University's Research Portal is retained by the author(s) and / or other copyright owners and it is a condition of accessing these publications that users recognise and abide by the legal requirements associated with these rights.

\section{Take down policy}

The Research Portal is Ulster University's institutional repository that provides access to Ulster's research outputs. Every effort has been made to ensure that content in the Research Portal does not infringe any person's rights, or applicable UK laws. If you discover content in the Research Portal that you believe breaches copyright or violates any law, please contact pure-support@ulster.ac.uk. 


\section{Precision Printing of Customized Cylindrical Capsules with Multifunctional Layers for Oral Drug Delivery}

Xuefeng $\mathrm{Li}^{+, *}$, Chunchen Zhang ${ }^{+, *}$, Shuting $\mathrm{Wu}^{+, *}$, Xing Chen ${ }^{+, *}$, John Mai ${ }^{\perp}$, Ming-Wei Chang ${ }^{7, \$^{*}}$

${ }^{\dagger}$ Key Laboratory for Biomedical Engineering of Education Ministry of China, Hangzhou, 310027 PR China

¥Zhejiang Province Key Laboratory of Cardio-Cerebral Vascular Detection Technology and Medicinal Effectiveness Appraisal, Hangzhou, 310027 PR China

${ }^{\perp}$ Alfred E. Mann Institute for Biomedical Engineering at the University of Southern California, Los Angeles 90007, California, USA

${ }^{\S}$ Nanotechnology and Integrated Bioengineering Centre, University of Ulster, Jordanstown Campus, Newtownabbey, BT37 OQB, Northern Ireland, UK.

\section{Corresponding author}

Dr. Ming-Wei Chang

*Email: m.chang@ulster.ac.uk; Tel: + 442895367142 


\section{ABSTRACT:}

Advances in personalized medicine will require custom drug formulations and delivery mechanisms. Herein, we demonstrate a new type of personalized capsule comprising of printed concentric cylindrical layers with each layer having a distinctive functional drug component. Poly $\varepsilon$-caprolactone (PCL) with paracetamol (APAP) and chlorpheniramine maleate $(\mathrm{CM})$, synergistic drugs commonly used to alleviate influenza symptoms, are printed as an inner layer and outer layer, respectively, via micro-scaled electrohydrodynamic (EHD) printing. Polyvinyl pyrrolidone (PVP) nanofibers are embedded as interlayers between the two printed PCL-drug layers using electrospinning (ES) techniques. The complete concentric cylindrical capsule with a 6 $\mathrm{mm}$ inner diameters and $15 \mathrm{~mm}$ length can be swallowed for oral drug delivery. After dissolution of the PVP interlayer, the capsule separates in two, with inner and outer capsules for continuous drug dosing and targeting. Imaging was achieved using a 3T MRI system which allowed temporal observations of the targeted release though the incorporation of nanoparticles $\left(\mathrm{Fe}_{3} \mathrm{O}_{4}\right)$. The morphology and structure, chemical composition, mechanical properties and biocompatibility of the capsules were studied in vitro. In summary, this new type of custom printed and electrospun capsule that enabled component separation, targeted drug release may advance personalized medicine via multi-drug oral delivery.

KEYWORDS: composite capsules; capsule separation, controlled release; micro printing; multicomponent drugs. 


\section{Introduction}

Compounding pharmacies and customized drug formulations have been advancing over the past few decades to meet demands of personalized medicine ${ }^{1-2}$. New medicine delivery systems have been invented which delivered drugs into the human body though the skin and nasal cavity ${ }^{3-4}$. However, oral formulations still remains the most widely used drugs delivery mechanism when dealing with treatment of chronic diseases and cancer therapy due to its convenience and efficacy ${ }^{5}$. Thus, developing methods to customize the release profiles of orally-administered drugs has become a research area that has attracted considerable interest ${ }^{6}$. A quick release profile supplies a sufficient dosage to deal with some acute conditions and a slow release profile delivers a steady dosage to treat chronic conditions while also decreasing the frequency of daily ingestion of pills ${ }^{7}$. Historically, the drug release profile was modified by changing the structure of the delivery capsule or modifying the concentration of excipients ${ }^{8-9}$. Also, multicomponent drugs were developed for improved targeting of disease inhibitors, which not only improves dosing convenience but also deals with complex disease comorbidities such as cardiovascular and cerebrovascular diseases synergistically ${ }^{10-12}$.

Traditionally, capsules are solid dosage forms in which the drug is enclosed within a soluble shell with diameters ranging from $5.06 \mathrm{~mm}$ to $8.15 \mathrm{~mm}$, and a capsule length between $12.1 \mathrm{~mm}$ and $19.8 \mathrm{~mm}$. Capsules have the advantage that they are easily administered, they can cover up unpleasant drug odors and taste, and dissolve rapidly ${ }^{13-}$ ${ }^{15}$. However, typically, capsules can only be loaded with compatible drugs and this limited the development of multicomponent medicines to a great extent ${ }^{16-17}$. 
Furthermore, these mass produced capsules generally had a uniform dosage and release profile although high concentrations of drugs, such as bromide and aspirin, may stimulate the gastric mucosa which may lead to adverse side effects ${ }^{18-19}$. For example, a proper concentration of phenytoin sodium can be applied to the treatment of arrhythmia. Whereas, the a high concentration of phenytoin sodium in blood plasma concentration may lead to toxic effects ${ }^{20}$. Therefore, a specific drug dose and release profile are needed for improved efficacy and to minimize side effects due to individual differences in patients.

3D printing has been applied to tissue regeneration, wound healing, organ scaffolding and in pharmacology duo to its convenience and commercial availability ${ }^{21-}$ ${ }^{24}$. Fused deposition modeling (FDM), a form of 3D printing, has been reported and applied to the manufacturing of pharmaceuticals to realize customized production in the treatment of many diseases ${ }^{25-26}$. However, the high temperature of the printing nozzle during operation is not compatible with many thermosensitive drug compounds. Also, precision control of the millimeter-scale-diameter filament used in FDM 3D printing remains challenging in terms of accurate dosage control and the resulting drug release behaviors. In contrast, Electrohydrodynamic (EHD) 3D printing has advantages in terms of having a higher controllable resolution (e.g. the diameter of the filament can be on the micrometer or even nanometer scale) and is unheated ${ }^{27-28} \cdot 3 \mathrm{D}$ microstructures with drugs or bioactive compounds can be prepared in an ambient environment and the process can be done in a single ste ${ }^{29}$. A range of various polymers with viscosities ranging from $1 \mathrm{mPa} \cdot \mathrm{s}$ to $\sim 10,000 \mathrm{mPa} \cdot \mathrm{s}$ can be used as the carrier suspension to form 
a small jet from the nozzle orifice via an applied electrical field force in order to achieve ultrahigh resolution structures ${ }^{30}$. The formation of filaments and their resulting deposition accuracy at these smaller sizes can be controlled by parameters such as the applied voltage, the working distance, and the interactions of the solution properties ${ }^{31-}$ ${ }^{32}$. In addition, the electrospinning (ES) technique has been applied widely to engineer micrometer and nanometer scale fibers for encapsulation applications and tissue engineering of scaffolds ${ }^{33-34}$. A non-woven mat containing random fiber orientations can be quickly fabricated by the ES technique.

In this work, a new type of cylindrical, multicomponent capsule was demonstrated which integrated EHD 3D printing and ES techniques for the first time. This capsule does not have the traditional capsule weakness of a burst release in the gastrointestinal tract after ingestion. Specific release profiles can be designed via selecting appropriate inert materials and patterning the various structures to increase the desired surface area. PCL was selected as the carrier material, with paracetamol (APAP) and chlorpheniramine maleate $(\mathrm{CM})$ as the active drugs embedded into these outer and inner layers, respectively. Polyvinyl pyrrolidone (PVP) fibers are randomly electrospun and embedded as an interlayer between the two printed PCL layers. Once the PVP interlayer dissolves, the inner layer and outer layer of PCL membrane separate. By tracking the motion of encapsulated $\mathrm{Fe}_{3} \mathrm{O}_{4}$ nanoparticles in the PCL fibers, one part of the separated capsule containing $\mathrm{Fe}_{3} \mathrm{O}_{4}$ nanoparticles can travel to a specific location and release drugs with the assistance of an external magnetic field in order to realize the targeted therapeutic delivery. A multicomponent drugs capsule was designed to separate in order 
to achieve that dosage control over each component drug after separation. To the best of our best knowledge, this is the first reported fabrication of a separating capsule which contains multicomponent drugs with different dosage profiles for oral delivery.

\section{MATERIALS AND METHODS}

\subsection{Materials.}

Poly $\varepsilon$-caprolactone (PCL, Mw=80000 g/mol) and polyvinyl pyrrolidone (PVP, $\mathrm{Mw}=1300000 \mathrm{~g} / \mathrm{mol}$ ) were obtained from Sigma-Aldrich (St Louis, USA.) Dichloromethane (DCM), dimethyl formamide (DMF), ethyl ethanol (EtOH) and phosphate buffered saline (PBS $\mathrm{pH}=7.4)$ were obtained from Sinopharm Chemical Reagent Co. Ltd (Beijing, China.) Chlophrnamine maleate (CM) and paracetamol (APAP) were purchased from Aladdin Biochemical Technology Co, Ltd (Shanghai, China.) Paracetamol (APAP) is a common non-steroidal drug for anti-inflammatory antipyretic treatment of symptoms of the influenza and toothache pain while chlorpheniramine maleate $(\mathrm{CM})$ is an antihistamine which is a $\mathrm{H} 1$ receptor antagonist and is used for the treatment of rhinitis, sternutation, and other symptoms of mucosa allergy. Both APAP and CM were selected as model drugs due to their synergistic effect on alleviating influenza symptoms. $\mathrm{Fe}_{3} \mathrm{O}_{4}$ nanoparticles were obtained from HWRK Chem (Beijing, China). Unless otherwise noted, all materials were used directly without any further purification and disposition.

\subsection{Solution preparation}


PCL was dissolved into DCM and DMF (93:7 v/v) to prepare PCL solution $(\mathrm{w} / \mathrm{v}=$ $22 \%$ ) via magnetic stirring for 3 hours and rested for 1 hour at an ambient temperature of $25{ }^{\circ} \mathrm{C}$ to dissolve completely without bubbles. $\mathrm{CM}(\mathrm{w} / \mathrm{w}=0.5 \%)$ and APAP $(\mathrm{w} / \mathrm{w}$ $=5 \%$ ) were dissolved into the PCL solution separately. PVP was dissolved into EtOH (18 wt\%) with magnetic stirring for 5 hours at a speed of $150 \mathrm{rpm} .1 \%$ (w/w) $\mathrm{Fe}_{3} \mathrm{O}_{4}$ nanoparticles were added into the PCL solution and magnetically stirred for another two hours.

\subsection{Fabrication of cylindrical capsule}

The cylindrical capsule has a $6 \mathrm{~mm}$ inner diameter and is $15 \mathrm{~mm}$ in length. It was fabricated by combining 3D printing and ES technology with a cylindrical collector. Both the inner layer and outer layer of the capsule were fabricated via EHD 3D printing using the same parameters. The process for capsule fabrication is shown in Figure 1. The EHD 3D printing system (Figure 1(a)), which includes a syringe pump, high voltage supply, X-Y motion translation platform and cylindrical collector, is used to fabricated the inner layer and outer layers of the cylindrical capsule. During EHD 3D printing, the flow rate was set at $0.3 \mathrm{ml} / \mathrm{h}$ and the voltage was set at $2.5-2.7 \mathrm{kV}$. The distance between the metal needle and the cylindrical collector was set at 3-4 mm. The rotation speed of cylindrical collector was set at $150 \mathrm{rpm}$ and the $\mathrm{X}$-axis moved at 6 $\mathrm{mm} / \mathrm{s}$ between two points whose distance was $15 \mathrm{~mm}$ apart and this was repeated 150 times. The interlayers were fabricated via electrospinning (Figure 1(b)) with the same equipment as used for EHD 3D printing at a flow rate of $0.8 \mathrm{ml} / \mathrm{h}$ for the PVP solution and at voltages from $8-10 \mathrm{kV}$. The distance between the metal needle was set at 10 - 
$15 \mathrm{~mm}$ and the cylindrical collector was rotating at $600 \mathrm{rpm}$. The fabrication of the complete cylindrical capsule, as illustrated in Figure 1(c) has a PVP interlayer that separates the PCL-APAP and PCL-CM drug layers.

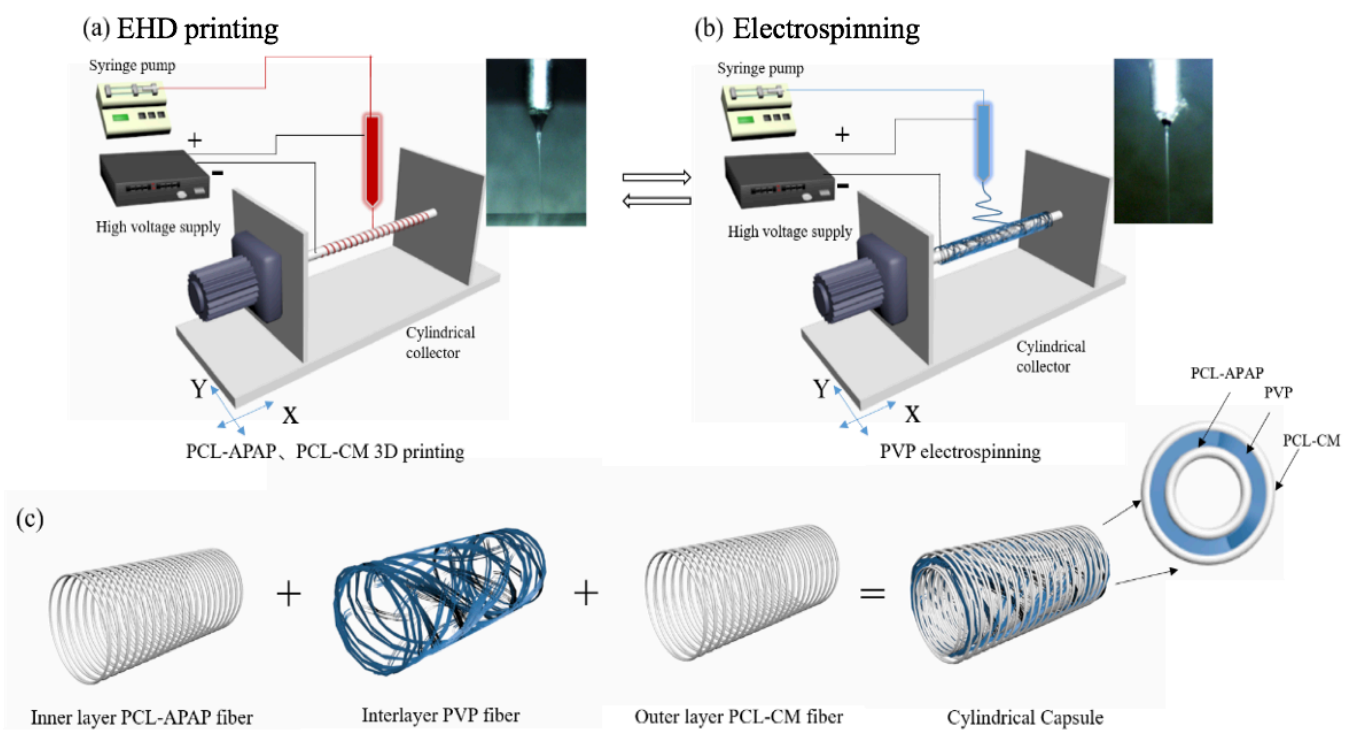

Figure 1. Schematic illustration of the experimental setup, fabrication process and structure of the composite layers rolled into concentric cylinders to fit inside an oral delivery capsule. The fabrication process includes (a) inner and outer PCL layers fabricated using EHD printing, (b) electrospinning of the PVP interlayer, and (c) the 3 fiber layers are combined into a composite concentric cylinder.

\subsection{Morphology characters of the Cylindrical capsule}

Optical microscopy (OM) (Pheonix BMC503-ICCF, China) and scanning electron microscopy (SEM) (S-4800, Hitachi, Japan) were used to examine the surface morphology of the fibers. Each sample was coated with a thin-film layer of gold via sputter coating for 3 minutes before SEM imaging. All the microsopy images were scaled using ImageJ software (National Institutes of Health, USA). 


\subsection{Fourier transform infra-red (FTIR) spectroscopy}

Fourier transform infra-red (FTIR) spectroscopy (Ir affinity 1, Shimadzu, Japan) was used to determine the chemical composition of the capsule via the $\mathrm{KBr}$ pellet pressing method. $2 \mathrm{mg}$ of each component (pure PCL, pure PVP, pure CM, pure APAP, PCL containing CM, PCL containing APAP and the whole capsule) was mixed with $200 \mathrm{mg}$ $\mathrm{KBr}$ and grinded down and compressed into transparent pellets under $12 \mathrm{MPa}$ of pressure. Then all the pellets were scanned with FTIR between $4000 \mathrm{~cm}^{-1}$ to $450 \mathrm{~cm}^{-1}$. Each spectrum was obtained from the average of 20 scans.

\subsection{Raman spectroscopy}

Raman spectroscopy and mapping was performed to characterize the distribution of the APAP and CM in the printing fibers. In situ printed fibers with 50 layers were evaluated using a laser confocal raman spectrometers (LabRAM HR Evolution, Horiba Jobin Yvon, France) using a $532 \mathrm{~nm}$ argon ion laser with a peak power of $300 \mathrm{~mW}$. Surface areas of $100 \mu \mathrm{m} * 100 \mu \mathrm{m}(\mathrm{X}-\mathrm{Y}$ plane) of the fibers were scanned with $20 \times 20$ pixels resolution in $\mathrm{X}$ and $\mathrm{Y}$ using a $50 \mathrm{X}$ objective $^{35}$. Spectra was scanned from 200 to $1800 \mathrm{~cm}^{-1}$ with $10 \mathrm{~s}$ exposure time and $1 \mathrm{~s}$ accumulation time. Labspace 6 software (Horiba, France) was used to generate false color maps with characteristic peaks of 454 $\mathrm{cm}^{-1}$ and $1258 \mathrm{~cm}^{-1}$ for APAP and $1094 \mathrm{~cm}^{-1}$ and $1594 \mathrm{~cm}^{-1}$ in wavenumber for CM in order to identify characteristic Raman peaks ${ }^{36-37}$.

\subsection{Differential Scanning Calorimetry}

Differential scanning calorimetry (DSC) tests were carried out to examine the thermal 
properties of the printed cylindrical capsules using a DSC 1/400 (METTLER-TOLEDO, Switzerland). DSC tests were performed under a nitrogen atmosphere. The capsule and its components underwent temperature changes ranging from $25^{\circ} \mathrm{C}$ to $260^{\circ} \mathrm{C}$ with a change rate set at $10^{\circ} \mathrm{C} / \mathrm{min}$.

\subsection{X-ray diffraction}

X-ray diffraction (XRD) tests were carried out with a X-ray diffractometer (Gemini A Ohra, Oxford, UK) to check the crystal texture of the capsule components. The capsule was scanned over a range from $5^{\circ}$ to $60^{\circ}$ over $2 \theta$ with step a change of $0.02^{\circ}$ at $40 \mathrm{~mA}$ and $40 \mathrm{kV}$.

\subsection{Magnetization curve test}

The magnetization curve of samples with different content of $\mathrm{Fe}_{3} \mathrm{O}_{4}$ nanoparticles was monitored via vibration sample magnetometer (Versalab, Quantum Design, Inc, San Diego, USA) at 300K under magnetic field strengths between -10000 Oe and 10000 Oe. This was done to obtain material properties for subsequent MRI analysis.

\subsection{In vitro MRI testing}

MRI images were obtained from a 3T MRI system (Magnetom prisma; Siemens Healthcare, Erlangen, Germany). Centrifuge tubes containing agar gel to mimic human body and capsule samples with different thicknesses and inner diameters were put into the head/neck coil region which has 20 channels. For agar gel preparation, 1g agar was mixed with $100 \mathrm{~mL}$ DI water, then heated to $100{ }^{\circ} \mathrm{C}$ and cooled. This temperature cycling was carried out several times to obtain a homogeneous agar gel. For the samples, 
cylindrical layers with diameters of $6 \mathrm{~mm}$ and $10 \mathrm{~mm}$, respectively, with $1 \% \mathrm{Fe}_{3} \mathrm{O}_{4}$ nanoparticles embedded in the outer and inner PCL layers were printed using the cylindrical collector. X-axis scans were repeat 150 and 300 times, with respect to the 6 $\mathrm{mm}$ and $10 \mathrm{~mm}$ cylindrical capsules, to investigate the effect of the dimensions of the PCL fibers on MRI imaging.

\subsection{Mechanical test}

Compressed mechanical tests were performed to identify the mechanical properties of the capsules with respect to different interlayer thicknesses and total cross-sectional area. This information is listed in Table 1. Each sample was separately tested under uniaxial compression along the longitudinal and radial axis. A universal material testing machine (Zwick Roell Z020, Zwick, Germany) was employed with a 500N mechanical sensor. Samples were compressed to $80 \%$ of either their original longitudinal or radial capsule dimensions, respectively, at a rate of $5 \mathrm{~mm} / \mathrm{min}$. Stress, $\sigma$, is calculated from the following equation 1 :

$$
\sigma=\frac{F}{S A}
$$

Where $\mathrm{F}$ is the applied compression force and $S A$ is the change in the cross-sectional area of the samples ${ }^{38}$.

Strain is calculated using equation 2:

$$
\varepsilon=\frac{\Delta L}{L_{0}}
$$

Where $\Delta L$ is the change in the length and $L_{0}$ is the original length of the sample.

The (Young's) modulus is obtained from the slope of the stress-strain curve in the linear region between $5 \%$ to $10 \%$ compression displacement with respect to the original 
size of the sample, as defined in equation 3:

$$
\text { Modulus }=\frac{\sigma}{\varepsilon}=\frac{F * L_{0}}{S A * \Delta L}
$$

Measurements for each sample were performed at least 3 times.

Table 1. Structural configuration of different samples used for testing of mechanical properties and for in vitro drug release.

\begin{tabular}{|c|c|c|c|}
\hline $\begin{array}{l}\text { Sample (cross- } \\
\text { sectional area } \\
\left.\text { mm }^{2}\right)\end{array}$ & Inner layer & interlayer & Outer layer \\
\hline S1 (9.06) & $\begin{array}{l}\text { PCL-APAP } \\
\text { fiber }\end{array}$ & -- & PCL-CM fiber \\
\hline S2 (20.87) & $\begin{array}{l}\text { PCL-APAP } \\
\text { fiber }\end{array}$ & $\begin{array}{c}\text { 1h electrospinning PVP } \\
\text { mat }\end{array}$ & PCL-CM fiber \\
\hline S3 (23.20) & $\begin{array}{l}\text { PCL-APAP } \\
\text { fiber }\end{array}$ & $\begin{array}{l}\text { 2.5h electrospinning } \\
\text { PVP mat }\end{array}$ & PCL-CM fiber \\
\hline S4 (26.38) & $\begin{array}{l}\text { PCL-APAP } \\
\text { fiber }\end{array}$ & $\begin{array}{l}\text { 2.5h electrospinning } \\
\text { PVP mat }\end{array}$ & $\begin{array}{l}\text { Double PCL- } \\
\text { CM fiber layers }\end{array}$ \\
\hline
\end{tabular}

\subsection{Separation tests}

Separation of the different cylindrical layers comprising the capsule is an important function of these multicomponent printed structures and has a direct impact on the individual drug release profiles. Separation tests were performed to identify the influence of the thickness of the electrospun interlayer and the rotation speed of the liquid environment on the separation efficiency. Four capsules, labeled from S1 to S4, with different thicknesses of the electrospun interlayer were obtained by changing the electrospinning times from $1 \mathrm{~h}$ (0.82 $\mathrm{mm}$ thickness $), 1.5 \mathrm{~h}$ (1.13 mm thickness), $2 \mathrm{~h}$ (1.41 $\mathrm{mm}$ thickness $)$ and $2.5 \mathrm{~h}$ (1.77 $\mathrm{mm}$ thickness). The four different capsules were immersed in $50 \mathrm{~mL}$ PBS solution at a stirring speed at $500 \mathrm{rpm}$ using a magnetic stirrer. 
In addition, capsules prepared at $1.5 \mathrm{~h}$ were examined at three other rotational speeds, specifically at $300 \mathrm{rpm}, 500 \mathrm{rpm}$, and $700 \mathrm{rpm}$, to characterize the separation time. The timer starts when the capsule was immersed into the PBS solution and ends with separation between the inner layer and outer layer of the capsule. Three capsule samples were test in each group.

\subsection{Interlayer mass loss test during separation}

In order to better understand the influence of the thickness of the interlayer on the separation, mass loss tests were carried out. Again, four groups of capsules were fabricated with different interlayer thicknesses $(1,1.5,2$ and 2.5h) and weighed $(M)$. Each capsule was immersed in a PBS solution with a stirrer rotating at $300 \mathrm{rpm}$. After a certain elapsed time $t i$ (where $t i=0.5,1,3,5$, and 10 min. respectively), the capsule was collected and dried. The mass is recorded as $\quad M t i$. Then, the dried capsule was immersed in DI water while stirring for 3 hours at $300 \mathrm{rpm}$ following by drying and weighed again. This mass is recorded as $\mathrm{M}_{0}$. The percentage of the mass loss during separation is calculated using equation 4 :

Percentage of the PVP mass loss $(\%)=\frac{M-M_{t i}}{M-M_{0}} * 100 \%$

$M-\mathrm{M}_{t i}$ is the mass after the PVP dissolved after a specific elapsed time $t i$. $M-M_{0} \quad$ is the mass after the PVP dissolved completely.

\subsection{Drug release test}

Drug release tests were performed to examined the drug release profiles from the various capsules. In order to mimic the gastrointestinal environment encountered by 
orally administered drugs, three different capsules with different thickness were each immersed in simulated gastric fluid $(\mathrm{pH}=1.7)$ and simulated intestinal fluid $(\mathrm{pH}=7.4)$, respectively. Each sample was immersed into $10 \mathrm{ml}$ of simulated gastric fluid and incubated at $37{ }^{\circ} \mathrm{C}$ in a HZ-8801K thermostatic oscillator (Taicang Science and Education Factory, China) with a 200 rpm shaking speed to simulate oral condition. 3 $\mathrm{ml}$ of simulated gastric fluid solution containing any dissolved capsule layers was collected and examined by ultraviolet (UV) spectra measurement (UV-2600 spectrophotometer, Shimadzu, Japan) at set intervals. Then, $3 \mathrm{~mL}$ of fresh simulated gastric fluid was added into the drug release solution. After 120 mins, any remnants of the capsule layers were transfer into $10 \mathrm{~mL}$ of simulated intestinal fluid solution and the above UV measurement steps were repeated. The UV absorption values of CM and APAP were selected at wavelengths of $243 \mathrm{~nm}$ and $262 \mathrm{~nm}$, respectively. Each group had three samples. The release rate of the drugs were calculated using equation 5:

$$
\text { Drugs release rate }(\%)=\frac{C t}{C M} * 100 \%
$$

$C t$ is the drug mass released from the capsule during an elapsed time t. $C M$ is the maximum mass released from the capsule.

In order to determine the drug release mechanism, the drugs release rate was fitted to several models such as zero-order, first-order, Higuchi, Korsmeyer-Peppas and HixsonCrowell models.

\subsection{Biocompatibility and cytotoxicity}

In order to determine the biocompatibility of the capsules, mouse fibroblast cells (L929) were used to test for cytotoxicity in vitro. MEM medium with 10\% FBS and 
$1 \%$ antibiotic-antimycotic solution were used to culture the $\mathrm{L} 929$ cells at $37^{\circ} \mathrm{C}$ in $5 \%$ $\mathrm{CO}_{2}$.

Before each experiment, all samples were sterilized under a UV light for $4 \mathrm{~h}$. Subsequently, the printed PCL-APAP fibers, the PCL-CM fibers and the capsule were added into the MEM medium at a concentration of $3 \mathrm{mg} / \mathrm{mL}$, and then added into a culture dish with $0.2 \mathrm{~mL}$ of $\mathrm{L} 929$ cells and $2 \mathrm{~mL}$ of MEM medium for 2 days.

After culturing for 2 days, L929 cells were fixed using 4\% paraformaldehyde and permeabilized using $0.1 \%$ Triton X-100. The cell cytoskeletons and nuclei were stained using Alexa Fluor 546 phalloidin and 4',6'-diamidino-2-phenylindole, respectively. The morphology and distribution of cells was imaged using a fluorescent microscope (Nikon Ti-S, Japan).

A CCK-8 assay was applied to quantify cytotoxicity of L929 cells after culturing with the components of the capsule. A $200 \mu \mathrm{L}$ cell suspension was added into a 96-well plate and cultured $7 \mathrm{~h}$ with $1 \mathrm{mg}$ of PCL-APAP fibers, $1 \mathrm{mg}$ of PCL-CM fibers and the capsule, respectively. Then, $20 \mu \mathrm{L}$ of CCK-8 solution was added into each well and cultured according to assay instructions. A microplate reader (Multiskan Go, Thermo Fisher Scientific, USA) was used to read the absorbance at $450 \mathrm{~nm}$. Cell viability was calculated using the following equation:

$$
\text { Cell viability }(\%)=\frac{A s-A b}{A c-A b} * 100 \%
$$

Where As is the absorbance of the sample groups, Ac is the absorbance of control group (containing L929 cells and the CCK-8 solution), and Ab is the absorbance of the blank group without samples, L929 cells and CCK-8 solution. 


\subsection{Statistical analysis}

All experiments were carried out at least three times and data is shown as the mean \pm standard deviation $(n \geq 3)$. Statistical analyses were performed using SPSS Statistical v18 software (IBM, UK.). If the result of Student's t-test is $* \mathrm{p}<0.05$, the statistical difference is determined to be statistically significant.

\section{Results and Discussion}

\subsection{Design and fabrication of capsules}

The capsule fabrication process and schematic illustrations of the completely assembled capsule are shown in Figure 1. Capsules with $6 \mathrm{~mm}$ outer diameters and $15 \mathrm{~mm}$ in length were designed and fabricated. The inner layer and outer layers of the capsule consisting of PCL-APAP fibers and PCL-CM fibers, respectively, were prepared using a stable cone jet during EHD 3D printing at voltage from $2.5-2.7 \mathrm{kV}$. The PVP interlayer was generated using a stable cone jet during electrospinning at applied voltages from 8-10 kV. Deposition of the PVP layers was fabricated at varying times of 1 hour, 1.5 hours, 2 hours and 2.5 hours. Figure 1c shows the integration process and a cross-sectional illustration of the complete capsule containing an inner layer of PCL-APAP fibers, an interlayer of PVP fibers and an outer layer of PCL-CM fibers.

Figure 2 shows the OM and SEM images of the capsule components generated using EHDP and ES methods. The arrangement and deposition of the inner layer PCL fibers 
are shown in Figure 2(a), 2(b) and 2(c). The printed fibers are well-aligned with fiber diameters ranging from $17.51 \pm 5.77 \mu \mathrm{m}$. Many holes can be observed on the surface of the PCL fibers in the high magnification SEM image (Figure 2(c)) due to solvent volatility $^{39}$. The electrospun interlayer of PVP fibers are shown in Figure 2(d), 2(e)) and 2(f). These fibers have uniform dimeters of $1.65 \pm 0.17 \mu \mathrm{m}$ and a smooth surface. OM and SEM images of the outer PCL-CM layer of the complete capsule are shown in Figure 2(g) and 2(h), respectively. The diameter of the outer layer (comprised of PCLCM fibers) is $20.35 \pm 7.76 \mu \mathrm{m}$ and is larger than the diameter of the inner layer (of PCLAPAP) fibers. This is due to the shorter distance between the needle and the fiber deposition area. The insert picture of Figure 2(h) presents a cross-section of the capsule which clearly shows the multi-layered deposition of the concentric cylindrical capsule containing an inner layer of PCL-APAP fibers, the interlayer of PVP fibers, and the outer layer of PCL-CM fibers. It is noted that the thicker interlayer thickness may affect the printing of the outer layer due to the change in the local electric field gradient. However, the whole process was stable during EHD printing and electrospinning, yielding a final capsule product as shown in Figure 2(i). 

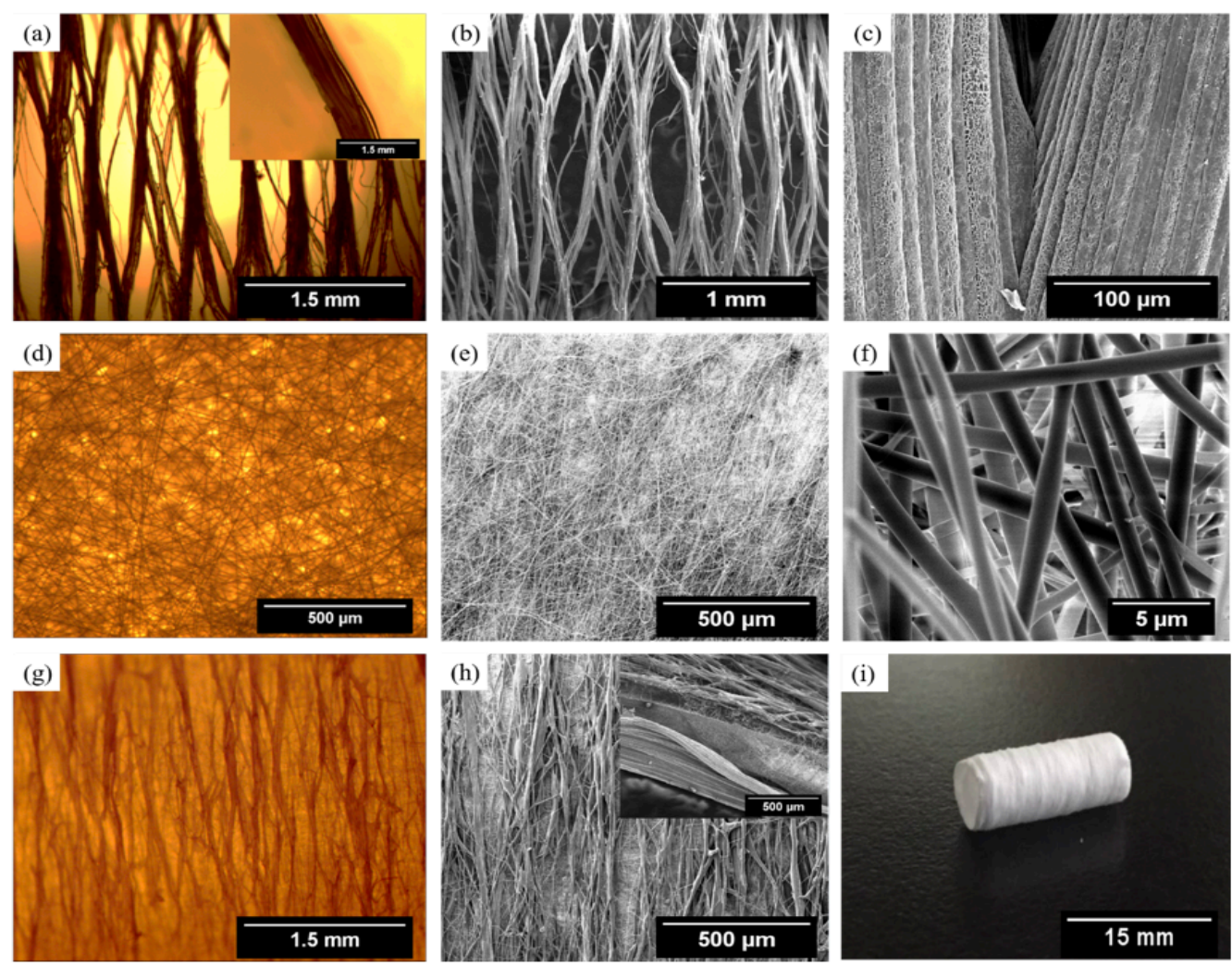

Figure 2. OM and SEM images of components and the integrated capsule. (a) OM images and (b) SEM image of the EHD printed PCL-APAP fibers and (c) fibers at higher magnification; (d) OM images and (e) SEM image of electrospinning PVP fibers and (f) fibers at higher magnification; (g) OM images and (h) SEM image of the EHD printed outer layer (PCL-CM) and a cross-sectional image of the integral capsule (inset); (i) Image of the complete capsule.

\subsection{Raman spectra results}

The distribution of drugs in the printed fibers was non-destructively examined via their Raman spectra ${ }^{40-41} .50$ layers of printed fibers of PCL-APAP and PCL-CM were tested. Figure 3 is a Raman mapping over a $100 * 100 \mu \mathrm{m}^{2}$ area of the PCL-APAP and PCL-CM fibers. The color in the map indicates the drug content. The change in color 
ranging from blue to red indicates the relative concentration of a drug. An OM image of the PCL-APAP fiber is presented in Figure 3(a), while false color mappings of the PCL-APAP fiber with characteristic Raman peaks for APAP ${ }^{37}$ at $1258 \mathrm{~cm}^{-1}$ and $458 \mathrm{~cm}^{-}$ ${ }^{1}$ are shown in Figure 3(b) and Figure 3(c), respectively. The change of intensity from blue to red in the mapping scale is mostly due to the spatial distribution of the different concentrations of the APAP present and the (blue) background area indicates PCL. The Raman spectrum shows that the APAP is distributed homogenously in the printed fibers. The yellow and green scope of region indicted the APAP may remain amorphous state ${ }^{41}$. However, OM (Figure 3(d)) image and Raman false color mapping of PCL-CM fiber with nearby character peaks $1094 \mathrm{~cm}^{-1}$ (Figure 3(e)) and $1594 \mathrm{~cm}^{-1}$ (Figure 3(f)) of CM${ }^{42}$. Similar result displaying the distribution of the CM (red, yellow and green regions) is presented in the false color mapping of the PCL-CM fiber. These results confirm the successful drug encapsulation in micro scaled printed fibers and their drugs distribution is homogeneous.
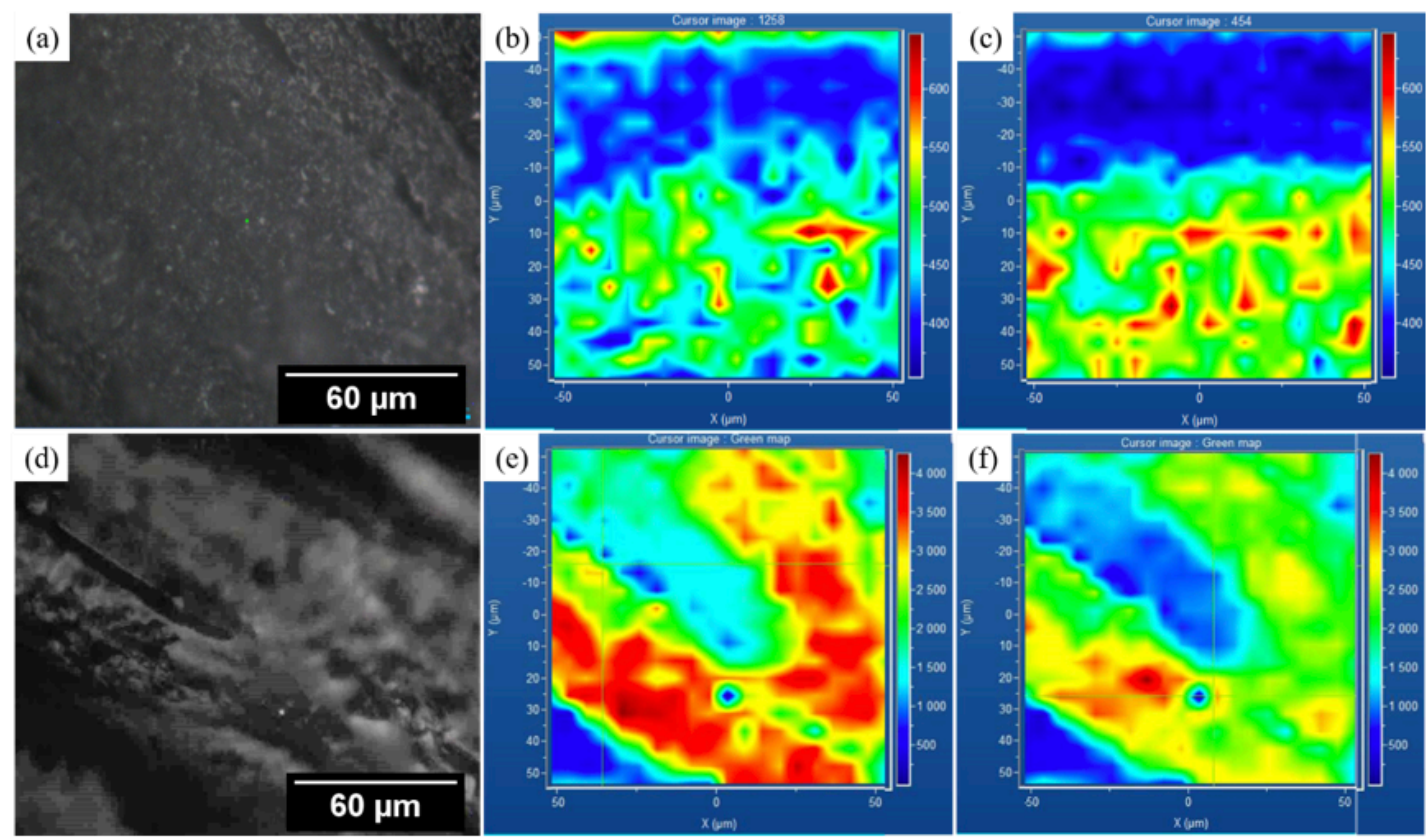
Figure 3. Raman false color mapping of PCL-APAP fibers and PCL-CM fibers. OM image (a) and false color mapping of PCL-APAP fibers with characteristic peaks at (b) $1258 \mathrm{~cm}^{-1}$ and (c) $454 \mathrm{~cm}^{-1}$ for APAP. OM image (d) and false color mapping of PCL-CM fibers with characteristic peaks at (e) $1094 \mathrm{~cm}^{-1}$ and (f) $1594 \mathrm{~cm}^{-1}$ of CM.

\subsection{Fourier transform infrared spectroscopy, DSC thermograms and XRD} analysis

FTIR spectroscopy was used to confirm the chemical composition of the capsule components before and after fabrication using printing and electrospinning. Figure 4(a) shows the FTIR spectra of pure PCL, pure PVP, pure APAP and pure CM. Pure PCL polymer peaks are observed at $1725 \mathrm{~cm}^{-1}$, which is due to carbonyl $(\mathrm{c}=0)$ groups ${ }^{43}$. Other peaks, such as at $2868 \mathrm{~cm}^{-1}$ and $2949 \mathrm{~cm}^{-1}$, arise from methylene $\left(\mathrm{CH}_{2}\right)$ groups. The presence of bands at $1643 \mathrm{~cm}^{-1}$ arise from $\mathrm{C}=\mathrm{O}$ groups, $1018 \mathrm{~cm}^{-1}$ and $1076 \mathrm{~cm}^{-1}$ are due to C-N stretching and the $\mathrm{N}-\mathrm{OH}$ complex at $1288 \mathrm{~cm}^{-1}$ confirm the existence of pure PVP and within the fabricated capsule ${ }^{44}$. For pure APAP, the characteristic peaks are present at $3200 \mathrm{~cm}^{-1}$ due to $\mathrm{OH}$ and $\mathrm{NH}$ stretching along with secondary bands at $1700 \mathrm{~cm}^{-1}$ and $1500 \mathrm{~cm}^{-145}$. For pure CM, the characteristic peaks at $3012 \mathrm{~cm}^{-1}$ are due to $\mathrm{C}-\mathrm{H}$ stretching. The peaks at $1205 \mathrm{~cm}^{-1}$ and $1151 \mathrm{~cm}^{-1}$ arises from $\mathrm{C}-\mathrm{N}$ vibration, and peaks at $1473 \mathrm{~cm}^{-1}$ are a result of C-C multiple bond stretching ${ }^{46}$. Figure $4 \mathrm{~b}$ is a FTIR spectrum of PCL-APAP, PCL-CM and the composite capsule. Some characteristic peaks for APAP and CM were present in the composite structure. This shows that the fabrication of the composite capsule with multicomponent drug layers was achieved via 
integration of EHD printing and electrospinning.
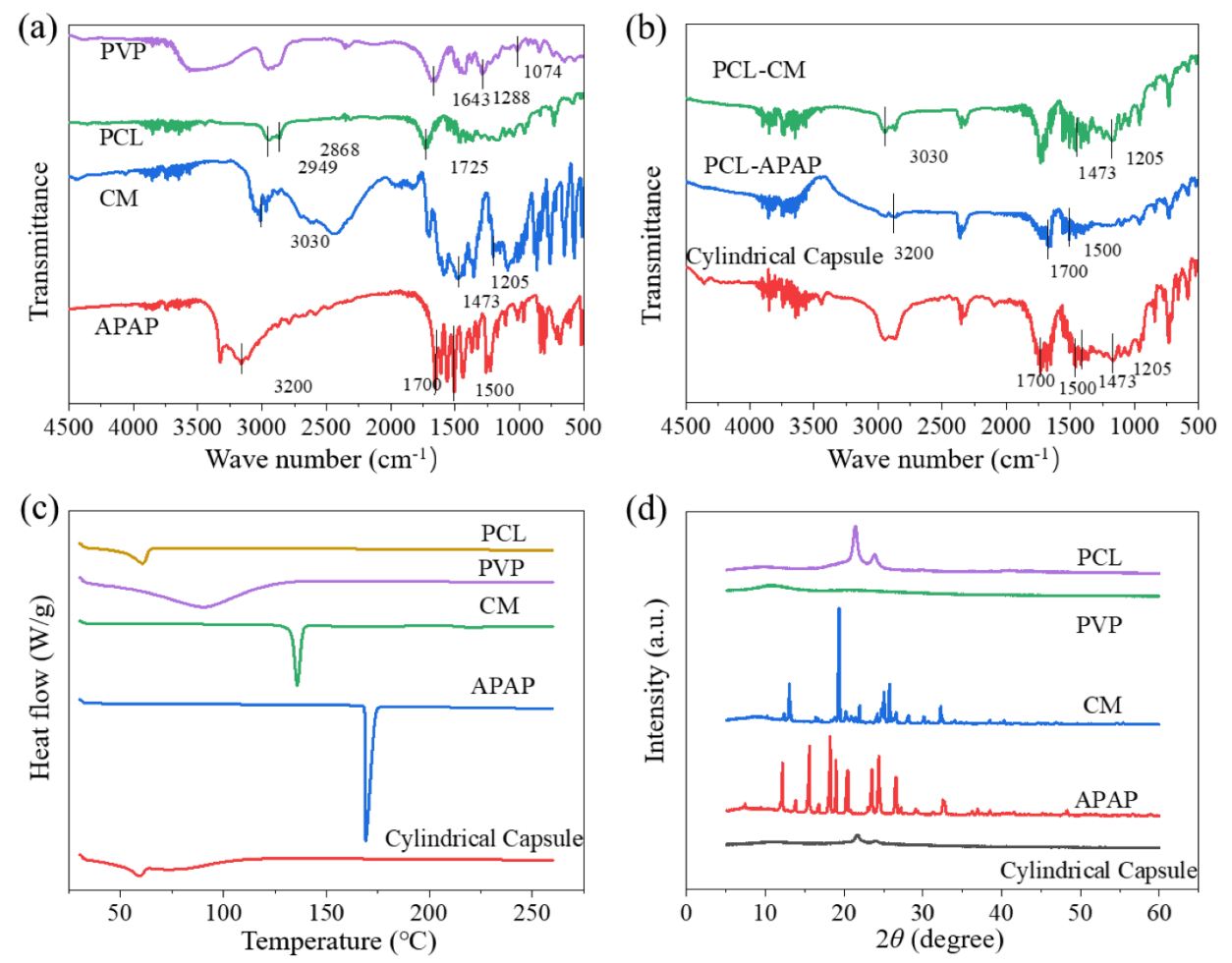

Figure 4. (a) FTIR spectrum of pure PCL, pure PVP, pure APAP and pure CM, (b) FTIR spectrum of the EHD printed PCL-APAP fibers and PCL-CM fibers, and the combined capsule, (c) DSC thermograms of graphing the individual raw materials and the composite capsule, (d) XRD results comparing the individual raw materials and the composite capsule.

Figure 4(c) presents DSC thermograms of the capsule components and the complete capsule. The glass transition temperature of PCL, APAP and $\mathrm{CM}$ are $57^{\circ} \mathrm{C}, 156^{\circ} \mathrm{C}$ and $130^{\circ} \mathrm{C}$, respectively. Comparing each component of the capsule to the complete capsule, the complete capsule only displays an endothermic peak for PCL but has no peaks corresponding to APAP and $\mathrm{CM}$ which confirms that these drugs remain in an amorphous state ${ }^{47-48}$.

XRD analysis was performed in order to check the physical structure of the capsule 
components before and after printing and electrospinning. Figure 4(d) presents the XRD curves for pure PCL, pure PVP, pure APAP, pure CM and the complete capsule. For pure PCL, two strong Bragg diffraction peaks are visible at angles $2 \theta=21.4^{\circ}$ and $23.8^{\circ}$ which is due to the diffraction of the (110) and (200) lattice planes of PCL ${ }^{49-50}$. The measured XRD pattern shows a wide signal for pure PVP which indicates an amorphous state ${ }^{51}$. The crystallinity of the CM is seen in its unique XRD pattern with several sharp peaks at $13^{\circ}, 19.3^{\circ}, 25^{\circ}$ and $25.7^{\circ 52-53}$. The APAP has two forms: a monoclinic form and orthorhombic form. The XRD pattern for the APAP shows several peaks with Bragg angles at $12.14^{\circ}, 15.58^{\circ}, 18.2^{\circ}, 23.52^{\circ}$ and $24.38^{\circ}$. These results show that the APAP used was in its monoclinic form because its orthorhombic form has another characteristic peak at Bragg angle of $24.03^{\circ}$, which is missing in this figure ${ }^{37,}$

${ }^{54}$. However, the complete capsule only has weak peaks for PCL without the prominent peaks for APAP and CM. This confirmed again that the APAP and CM were in amorphous state after complete capsule fabrication. It should be noted that many crystalline drug compounds are poorly water-soluble and hence have non-ideal bioabsorption profiles. Amorphous forms of a drug exist at a higher energy state with increased molecular mobility compared to its crystalline form which is desirable for increasing the rate of bioabsorption into a tissue ${ }^{55}$. In addition, the amorphous state has advantages of higher solubility and chemical stability after mixing with polymers for therapeutic delivery ${ }^{56}$.

\subsection{Magnetization curves and MRI imaging}


In order to physically track the behavior of the complete capsule in situ, $\mathrm{Fe}_{3} \mathrm{O}_{4}$ nanoparticles were introduced into the PCL layers of the capsule. This allows for multifunctional uses of the capsule such as for targeted drug release using an externally located magnet, and as a magnetic resonance tracer. Figure 5(a) displays the magnetization curves of the complete capsules with different $\mathrm{Fe}_{3} \mathrm{O}_{4}$ nanoparticle concentrations. All these "s" curves pass through the graph origin point without hysteresis. As the applied magnetic field increases, the magnetic moment (Y-axis) of the complete capsule also increases. As expected, the magnetic moment increased with increased concentration of $\mathrm{Fe}_{3} \mathrm{O}_{4}$ nanoparticles which means that increasing the mass of $\mathrm{Fe}_{3} \mathrm{O}_{4}$ nanoparticles will improve the magnetic response to external magnetic fields.

Figure $5(\mathrm{~b})$ to $5(\mathrm{~g})$ show the capsule preparation for $\mathrm{T} 1$ graphing and $\mathrm{T} 2$ imaging using the MRI system. Capsules with different diameters $(6 \mathrm{~mm}$ and $10 \mathrm{~mm})$ and PCL layer thicknesses (by repeated printing 150 and 300 times, respectively) and containing $1 \% \mathrm{Fe}_{3} \mathrm{O}_{4}$ nanoparticles in the outer and the inner PCL layers were prepared to investigate the effect of the diameter and thickness of these PCL fiber capsules on MRI imaging. The outline of the complete capsule can be observed in the MRI system and more details are observed by increasing the thickness (printing times) of the capsule. However, no major difference can be observed in the MRI image when the capsule diameter is increased from $6 \mathrm{~mm}$ to $10 \mathrm{~mm}$. This can be due to the resolution of the 3T MRI system. Nevertheless, the basic capsule configuration used for drug delivery in this paper is also sufficient to be used as a magnetic resonance imaging tracer. 

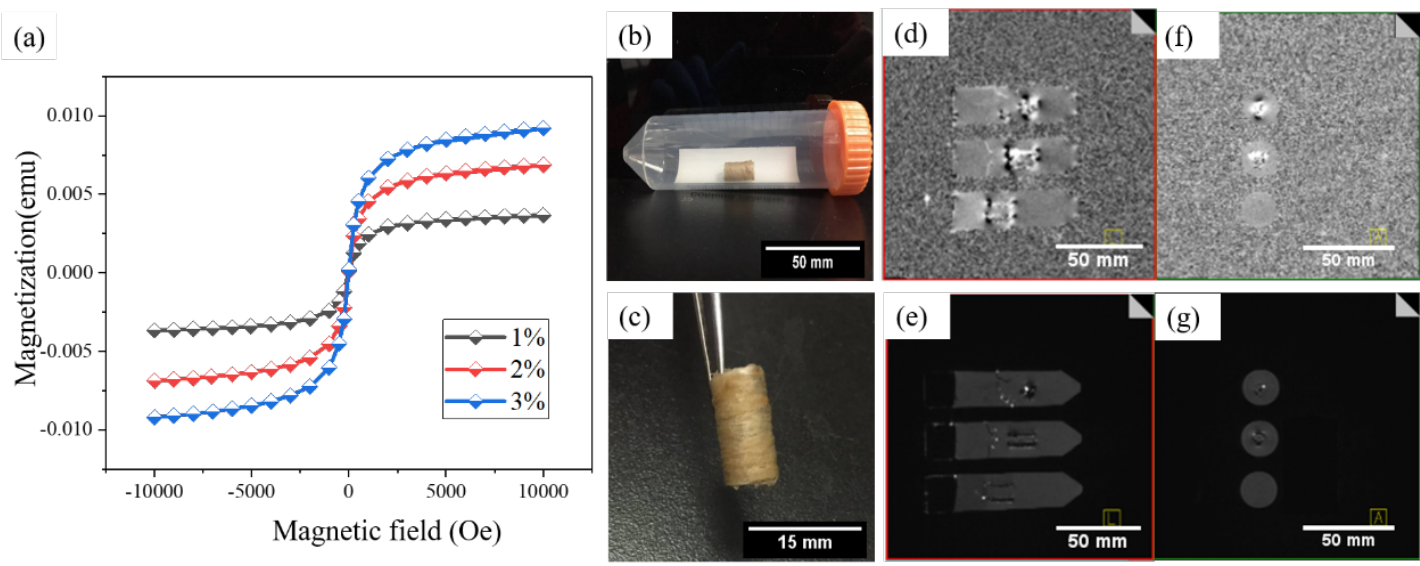

Figure 5. (a) Magnetization curves for the complete capsules with different $\mathrm{Fe}_{3} \mathrm{O}_{4}$ nanoparticles concentrations: $1 \%, 2 \%$ and $3 \%$ of the PCL mass. (b) and (c) are images of the capsule sample prepared for MRI testing. (d) and (f) are different views from T1 imaging: the three samples from top to bottom have a diameter of $6 \mathrm{~mm}$ with 150 times printing, a diameter of $6 \mathrm{~mm}$ with 300 times printing, and a diameter of $10 \mathrm{~mm}$ with 300 times printing, respectively. (e) and (g) are different views of T2 imaging: the three samples show, from top to bottom, have a diameter of $6 \mathrm{~mm}$ with 150 times printing, a diameter of $6 \mathrm{~mm}$ with 300 times printing, and a diameter $10 \mathrm{~mm}$ with 300 times printing.

\subsection{Mechanical test}

Figure 6 shows the complete capsule compressed in different directions and the corresponding measured mechanical properties. The sample parameters are listed in Table 1. Mechanical tests were performed in both the longitudinal and radial direction of the capsules as shown in Figure 6(a) and (d). Tests in the longitudinal direction confirms that the Young's modulus increased with an increase in either the printed PCL 
or electrospun PVP fibers (Figure 6(b)). The result in the radial direction test shows the same trend. However, as shown in Figure 6(e) the modulus of sample S1 is obviously higher than the other samples during testing in the radial direction because the crosssectional surface area of sample $\mathrm{S} 1$ is less than the other samples. This is consistent with Equation 3 where the cross sectional area affects the stress calculation and the resulting modulus. Furthermore, the PVP interlayer of the cylindrical capsule may reduce the strength of the capsule during radial-direction compressed test since the PVP polymer has weaker mechanical properties when compare to the PCL polymer. However, the tests in the longitudinal direction test do not show the above result, as seen in Figure 6(b) and the rate of change of the stress in the radial direction is lower than in the longitudinal direction during the first $10 \%$ of compression. During longitudinal compression, the thickness of the interlayer PVP effectively increased, and the capsule eventually failed due to buckling because of the lower material strength of the PVP.

(a)

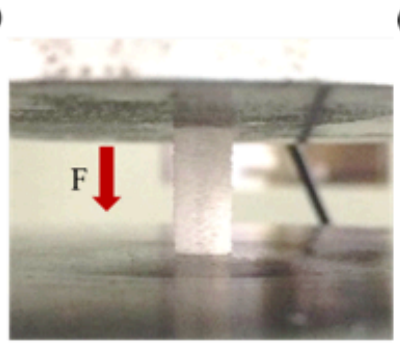

(d)

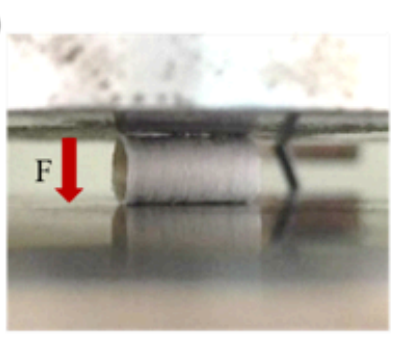

(b)
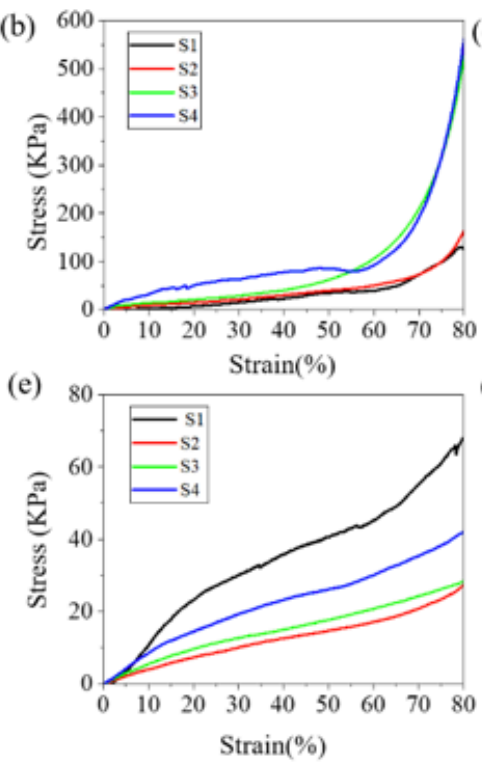

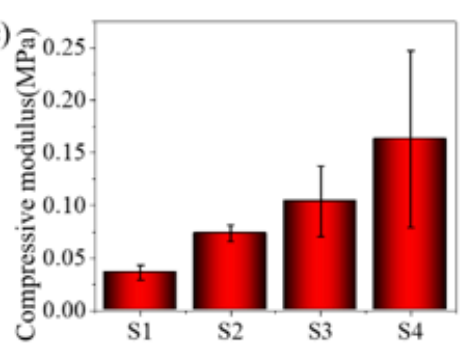

(f)

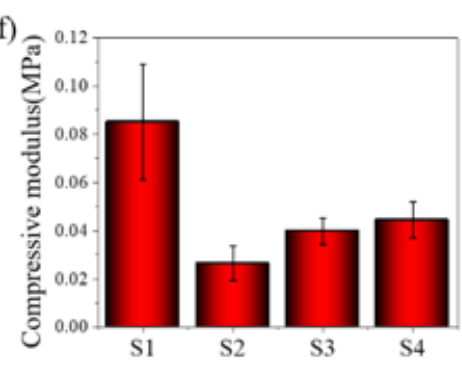

Figure 6. Mechanical testing of the complete capsules. (a) Photographs of capsules 
during compressive tests in the longitudinal direction. (b) Calculated stress-strain curves in the longitudinal direction for each sample capsule. (c) The Young's modulus due to longitudinal compression of each sample. (d) Pictures of a capsule during compressive testing in the radial direction. (e) Stress-strain curves for each sample under compressive testing in the radial direction. (f) Compressive modulus for each sample in the radial direction.

\subsection{In vitro separation process}

This separating composite capsule has the inherent ability to precisely control the release of each component drug. In order to verify this capability, in vitro testing was performed to observe separation of a composite capsule with a PVP interlayer that was ES for $2.5 \mathrm{~h}$ yielding an approximately $1.7 \mathrm{~mm}$ thickness. Figure 7 (a) shows the separation process for sample S3 at different time intervals from $0 \mathrm{~s}$ to $295 \mathrm{~s}$. The capsule sample was immersed in a $100 \mathrm{ml}$ beaker containing $50 \mathrm{~mL}$ PBS solution while being magnetically stirred at $100 \mathrm{rpm}$. The whole separation process was finished in $169 \mathrm{~s}$ and the complete dissolution of the PVP finished at $295 \mathrm{~s}$. A time-elapsed video of the separation pictured in Figure 7(a) is available as Video S1. It was interesting that during the separation process, the PVP did not dissolve from the capsule slowly, but the bulk of the PVP sheet rapidly separated from the capsule. This might be due to the centrifugal force on the PVP interlayer after water absorption. When there was enough space between the inner layer and outer layer, the PVP interlayer was ejected from the capsule and then the inner layer and outer layer successfully separated. 
Regulation and controlled movement of each component drug layer after separation was achieved by adding $\mathrm{Fe}_{3} \mathrm{O}_{4}$ nanoparticles and an external magnetic field. The magnetic response of these embedded nanoparticles provided a powerful method to control the drug release and drug tracking with MRI. ${ }^{57-58}$ Figure $7(\mathrm{~b})$ shows the process of magnetic attraction after two layer separation. The separated capsule containing the printed outer PCL with embedded $\mathrm{Fe}_{3} \mathrm{O}_{4}$ nanoparticles can be seen travelling quickly from the left to right sides of the beaker under an applied external magnet field, in a beaker containing $30 \mathrm{~mL}$ PBS solution, as shown in Figure 7(b) and Video S2. Note that the inner layer without any nanoparticles remained stationary. This travel distance was covered within $3 \mathrm{~s}$ at a velocity of $6.7 \mathrm{~mm} / \mathrm{s}$. Thus, starting with a single composite capsule, after capsule layer separation another new method for targeted delivery has been demonstrated via an applied external magnetic field.

(a)

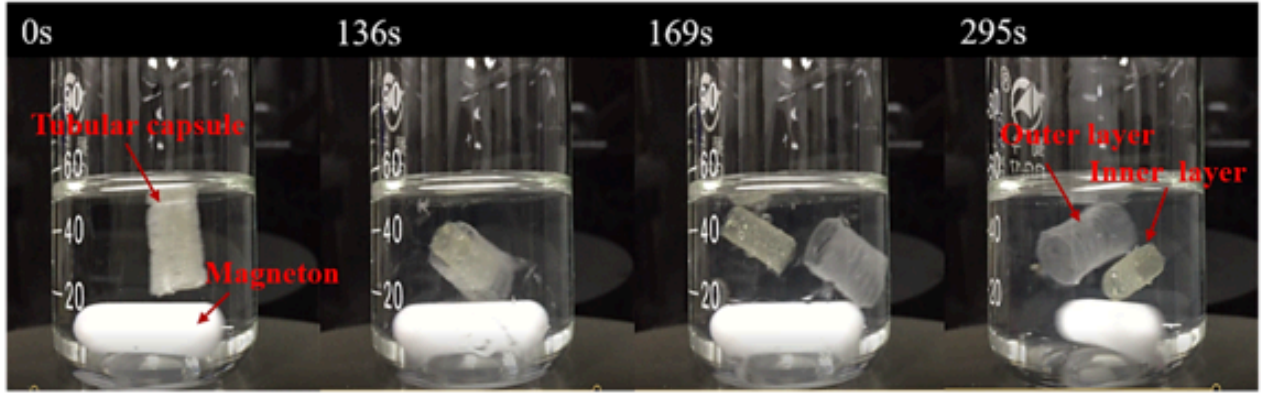

(b)

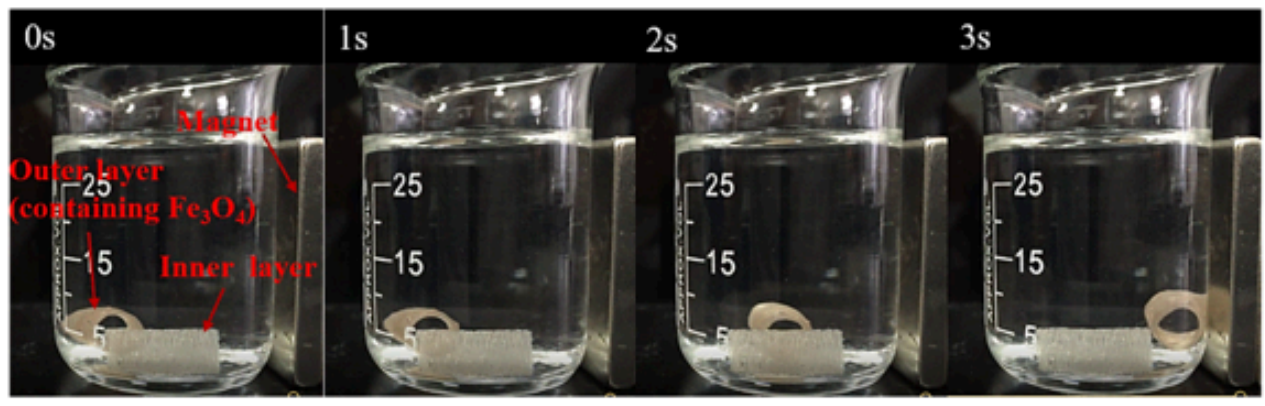

Figure 7. Separation process (a) of the composite cylindrical capsule (around $1.7 \mathrm{~mm}$ thickness) with a stirring speed at $100 \mathrm{rpm}$ in 50ml PBS solution and the magnetic-field 
induced translation process (b) outer layer containing nanoparticles travels from left to right in a $30 \mathrm{ml}$ PBS solution.

In order to further investigate the effect of other variables on capsule separation, the effects of electrospun PVP thickness and the stirring speed were tested. Figure 8(a) and 8(b) show the time required for capsule separation with respect to various electrospinning time for the PVP interlayer $(1 \mathrm{~h}, 1.5 \mathrm{~h}, 2 \mathrm{~h}, 2.5 \mathrm{~h})$ and different stirrer rotational speeds $(300 \mathrm{rpm}, 500 \mathrm{rpm}$ and $700 \mathrm{rpm})$ mimicking the gastrointestinal motility, respectively. The results indicated that the separation time decreased as the thickness of ES interlayer increased and the stirrer speed increased. Increasing the PVP mass did not increase the time required for separation but decrease the dissolution time, which was incongruent with our expectation. To confirm this result, loss of PVP interlayer mass was recorded during the capsule separation process and is graphed in Figure 8(c). These result show that the entire PVP layer disappeared from the capsule within almost $10 \mathrm{~min}$. For three different ES times to prepare the PVP interlayer (1.5h, 2h, 2.5h), more than $90 \%$ of the PVP mass was lost from the capsule in $3 \mathrm{~min}$. The rate of mass loss of the PVP from the capsule increased with increasing PVP electrospinning time. Although the mass of the PVP increased $(21.48 \pm 5.1,34.32 \pm 4.9,44.56 \pm 9.1$, $54.56 \pm 10.8 \mathrm{mg}$ ), the effect of this increasing mass on the dissolution time could be neglected arising from the huge surface areas of electrospinning fibers and assuming perfect water absorption by the PVP. However, the increased thickness of the PVP interlayer that accompanied any mass increasing can provide more space between the 
inn layer and outer layer for the PVP to dissolve due to increasing the wettable surface area. The whole separation time included the time for the PVP to dissolve between the two PCL fiber layers. Meanwhile, the interaction between the two PCL fiber layers played an important role during the separation process especially when the thickness of the PVP interlayer is small. Therefore, increasing the mass of the PVP interlayer would not prolong the dissolution and separation times, but the increasing the thickness of PVP interlayer could decrease any electrostatic interaction forces between the inner PCL-APAP layer and outer PCL-CM layer. 

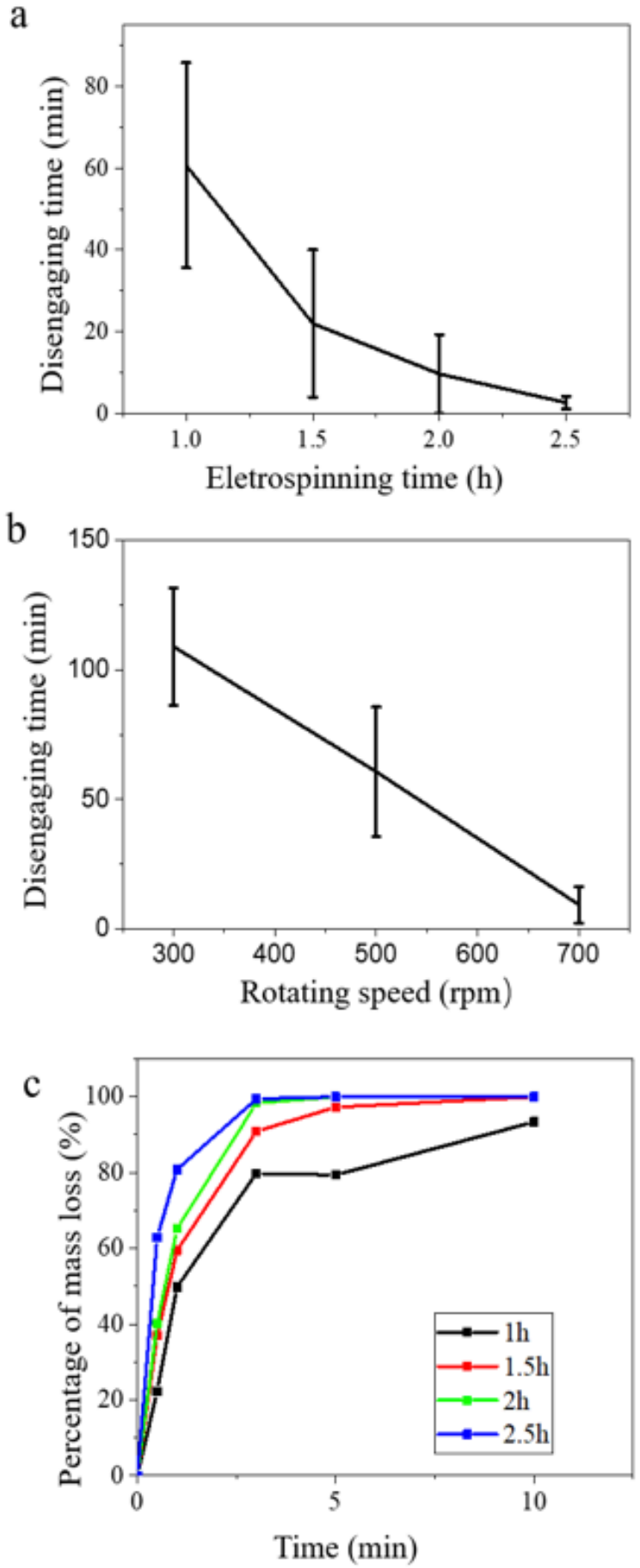

Figure 8. (a) Graph of dissolution time with different electrospinning time (1h, 1.5h, 2, 2.5h). (b) Graph of dissolution time with various rotating speed of magneton (300 rpm, $500 \mathrm{rpm}, 700 \mathrm{rpm}$ ). (c) Percentage of PVP mass loss in the four sample groups with different electrospinning times. 


\subsection{In vitro release of drugs}

The drug release profile of the APAP and CM from the various composite capsules was investigated in a simulated gastric fluid $(\mathrm{pH}=1.7)$ and simulated intestinal fluid $(\mathrm{pH}=7.4)$ at $37^{\circ} \mathrm{C}$ temperature to mimic conditions within the human body. Sample $\mathrm{S} 1$, S2 and S3 were selected to identify the influence of the interlayer thickness on the drugs' release profile. Each test was carried out in triplicate. The drug release profiles are plotted in Figure 9. The release profile of APAP was generally faster than CM. The whole release profile went through a fast release period in the first $30 \mathrm{~min}$ and then a slow release period. The fast release period could be attributed to the drugs being attached to the surface of the fibers, as showing in the Raman false color mapping. Furthermore, the porous structure on the surface of the PCL fibers, as seen on the SEM images, increased the specific surface area which increased the area for interaction with the drugs ${ }^{59-60}$.

Figure 9(b) presents the release profile of APAP and CM in the first 2 hours in detail. The tiny difference in release characteristics between these two drugs arise from differences in the cylinder structures. The release of APAP was faster than CM during the first 2 hours. The cumulative release of APAP was more than $90 \%$ while the cumulative release of $\mathrm{CM}$ was around $70 \%$. This may be that the APAP dissolves more easily than CM in an acidic condition. For the release profile of APAP loaded into the inner layer PCL fibers in different capsule structures, the increasing interlayer thickness increased the release rate possibly due to more space provided for drugs to diffuse into the liquid environment. The difference in interlayer thicknesses seemed to greatly 
influence the release rate of the CM. However, above a certain ES thickness, the release rate of the drugs was not faster. This might be related to the fiber diameters since the diameter of the PCL-CM fiber was larger than PCL-APAP fiber, on average.

The measured release rates were fitted to zero-order, first-order, the Higuchi, the Korsmeyer-Peppas and the Hixson-Crowell models to determine the mechanism of drug release. The results of the fitting parameters with regression coefficients and $n$ value are shown in Table 2 . The regression coefficients from modeling of the APAP reflects that the release process is more suitable to a first-order model and a Higuchi model, which describes the release profile of water-soluble pharmaceuticals in porous fibers ${ }^{61}$. The sample S1 without an interlayer was better fitted to a zero model and a first-order model. For CM, the Higuchi model was the best fit. As for the KorsmeyerPeppas model, it is not suitable in this situation because the cumulative drugs release reached and surpassed $60 \%$ in a short time. This result reflected that the appreciable quantity of drugs attached to the surface of the fibers which dissolved quickly in the liquid environment within the first several minutes. Therefore, the best fit for the drug release profiles for both APAP and CM from the composite capsules is the Higuchi model. In this work, two types of drugs can be encapsulated into the different components of the capsule and whose release rates can be controlled by the capsule thickness and the polymer combination. Also, the different release rates of the drugs are affected by the $\mathrm{pH}$ values of the gastrointestinal fluids. Thus, fast or long-term sustained release can simultaneously be achieved for acute treatment of symptoms while also decreasing the total number of daily doses. 
(a)

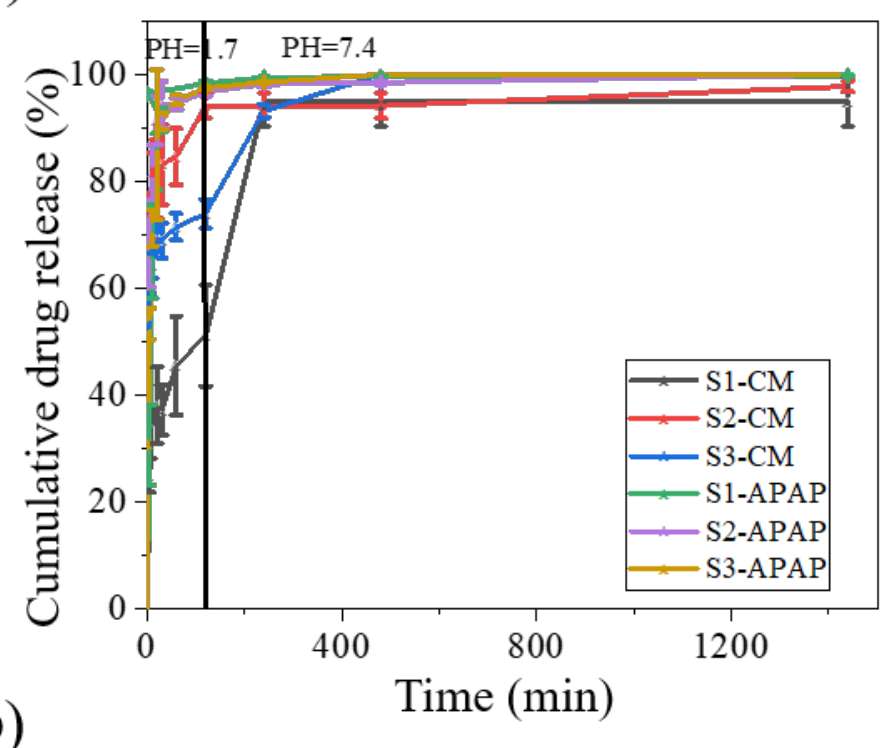

(b)

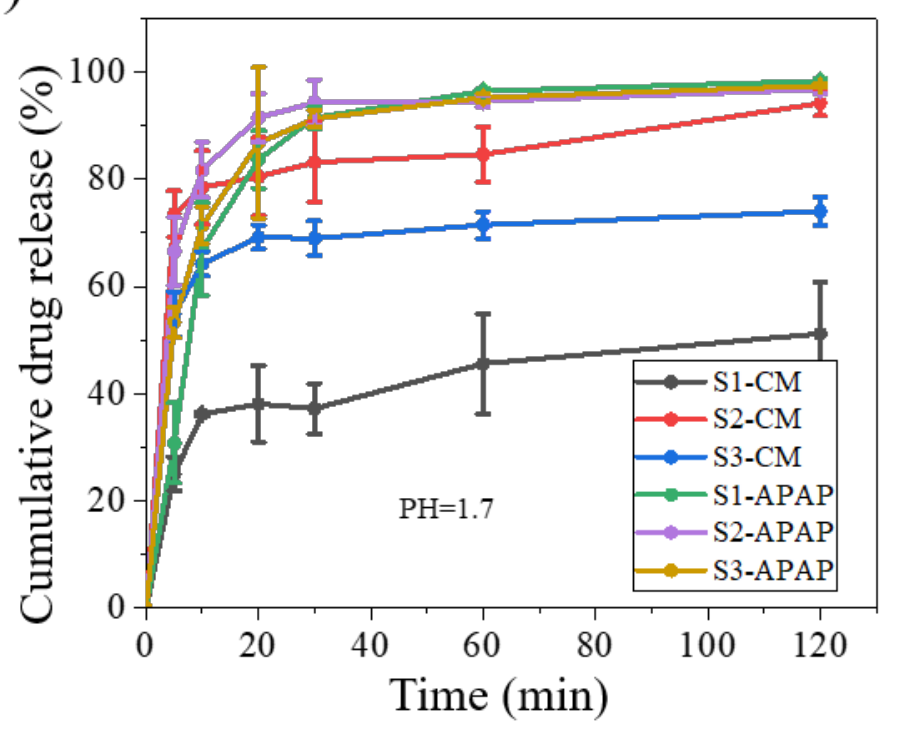

Figure 9. (a) Drugs release profile of APAP and CM from the various capsule configurations in a simulated gastric fluid $(\mathrm{pH}=1.7)$ and a simulated intestinal fluid ( $\mathrm{pH}=7.4)$. (b) Detailed drug release profile for APAP and CM from the composite capsule in the first two hours in a simulated gastric fluid $(\mathrm{pH}=1.7)$.

Table 2. Fitting Parameters for APAP and CM in the first $80 \%$ of drug release from the capsule with respect to several models. 


\begin{tabular}{cccccc}
\hline Drugs & Zero- & First- & Higuchi & Korsmeyer- & n \\
& order $\left(\mathbf{R}^{2}\right)$ & order $\left(\mathbf{R}^{2}\right)$ & $\left(\mathbf{R}^{2}\right)$ & Peppas $\left(\mathbf{R}^{2}\right)$ & value \\
\hline S1-APAP & 0.99 & 0.96 & 0.92 & 0.76 & 0.01 \\
S2-APAP & 0.88 & 0.97 & 0.99 & - & - \\
S3-APAP & 0.93 & 0.98 & 0.99 & - & - \\
S1-CM & 0.91 & 0.97 & 0.99 & - & - \\
S2-CM & 0.79 & 0.83 & 0.94 & - & - \\
S3-CM & 0.85 & 0.90 & 0.98 & - & - \\
\hline
\end{tabular}

\subsection{Biocompatibility}

Test for biocompatibility were carried out to identify the practical use of the composite capsule for oral delivery. L929 cells were cultured with PCL-APAP fibers, PCL-CM fibers and a partial capsule, respectively. Results are shown in Figure 10(a) with blue stains showing the live cell nucleus and the red cytoskeleton and indicates good biocompatibility. The L929 cells adhered to the fibers and maintained their morphology. Compared to cells adhering to PCL-APAP fibers, the number of cells sticking to the PCL-CM fibers was less and the morphology of these cells was not very plump, which was attributed to the cytotoxicity of $\mathrm{CM}^{62}$. Cytotoxicity of the capsule and its components were carried out and this result is shown in Figure 9(b). The results show cell proliferation after adding capsules into the L929 cells culture dish. Meanwhile, cell culturing showed that cell viability with PCL-CM fibers was lower than with other capsule components. Thus CM had an effect on cell proliferation, and 
the dose of CM in the capsule should to be decreased or limited.
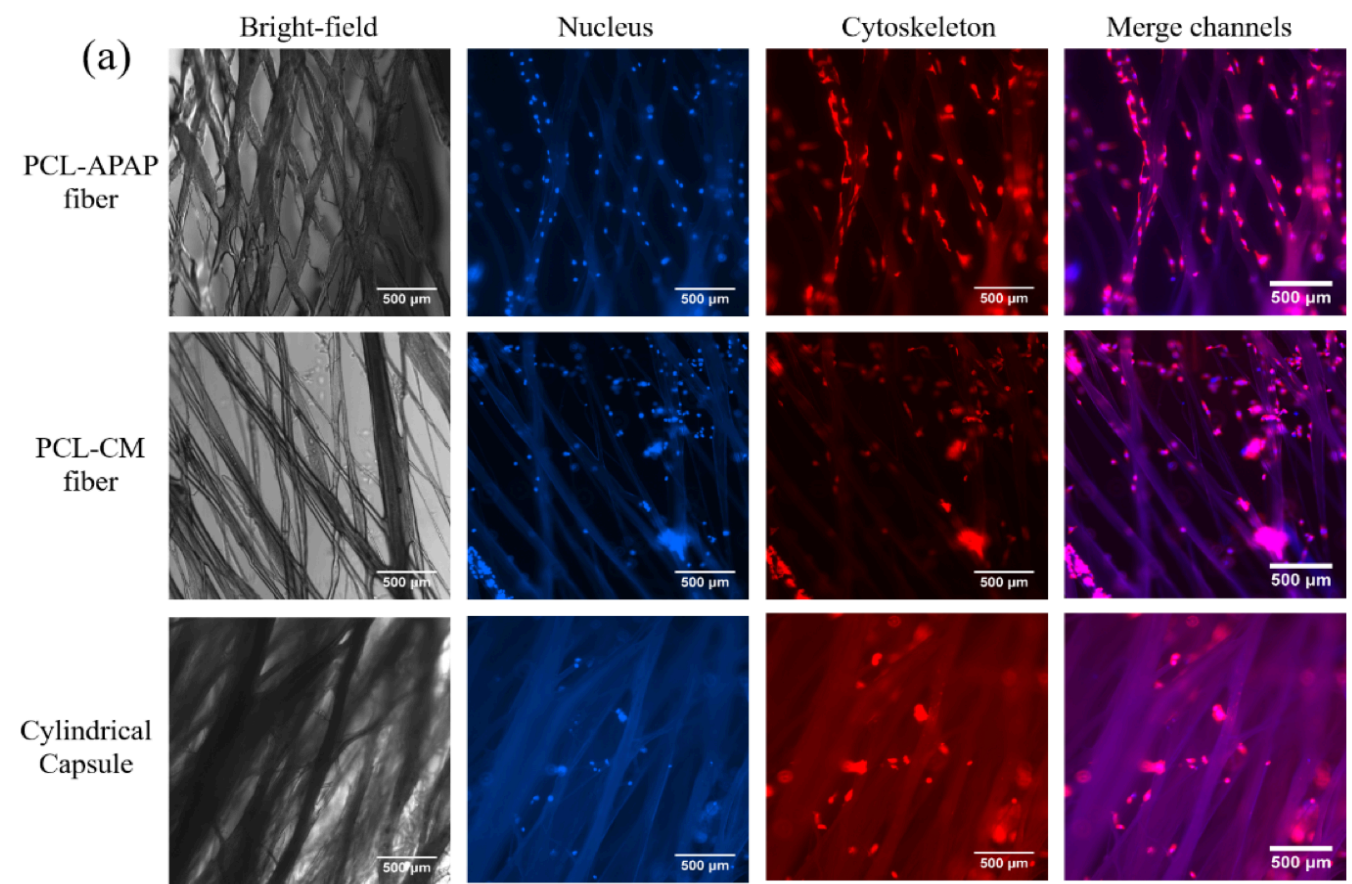

(b)

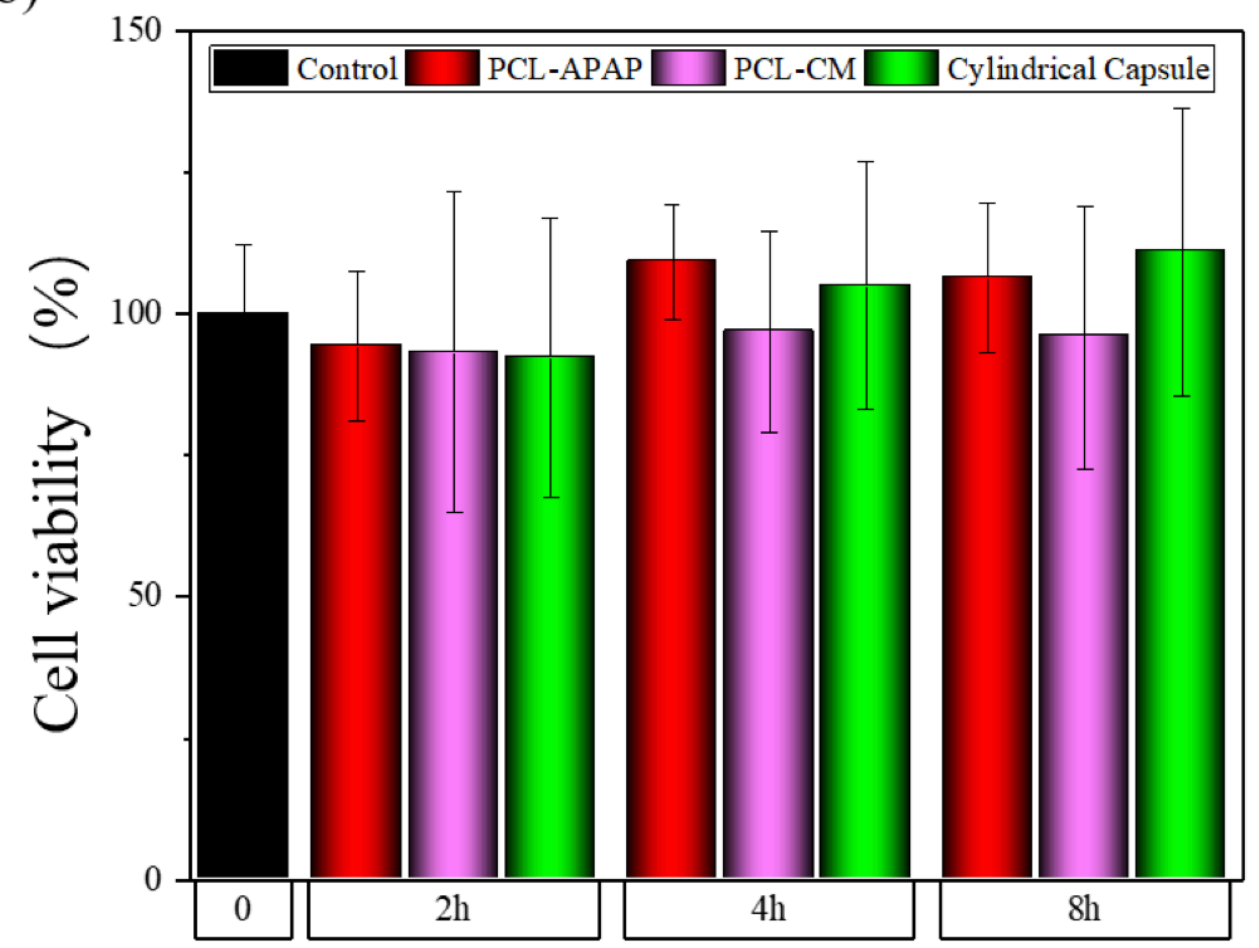

Figure 10. (a) Fluorescent micrographs of L929 cells cultured with capsule components PCL-APAP fibers and PCL-CM fibers and a partial capsule, respectively, after 2 days. (b) Cytotoxicity results with respect to each capsule components and the 
cylindrical capsule.

\section{CONCLUSIONS}

In summary, a separating oral capsule comprised of multicomponent fibers loaded with synergistic drugs was designed and fabricated via a combined EHD 3D printing and ES process. The capsule structurally is comprised of three concentric cylindrical layers: an inner layer of PCL-APAP fibers that were EHD printed, an interlayer of PVP that was electrospun and an outer layer of PCL-CM fibers that were EHD printed. Two different drugs, APAP and CM in amorphous form, are loaded into the PCL layers. Mechanical test indicts that capsule possess well mechanical properties. The two PCL fiber layers successfully separated after the PVP interlayer dissolved. Increasing the PVP interlayer thickness should promote quicker separation of the inner layer and outer layer. The incorporation of $\mathrm{Fe}_{3} \mathrm{O}_{4}$ nanoparticles provides an opportunity for targeted release of the separate cylinders and for MRI imaging applications. In vitro testing of the release of the two drugs indicts that the release of APAP and CM from the porous fibers mostly fit the Higuchi model and this composite capsule is biocompatible based on tests with L929 cell cultures.

\section{AUTHOR INFORMATION}

\section{Corresponding Author}

*Email: m.chang@ulster.ac.uk. Tel.: +44 2895367142 


\section{Author Contributions}

The manuscript was written through contributions of all authors. All authors have given approval to the final version of the manuscript.

\section{Notes}

The authors declare no competing financial interest.

\section{ACKNOWLEDGMENT}

This work was financially supported by the National Natural Science Foundation of China (No.81771960), the Key Science Technologies R\&D Program of Zhejiang Province (2015C02035) and the Fundamental Research Funds for the Central Universities (2017QNA5017).

\section{Supporting Information}

Supporting Information available:

- Separation process of the composite cylindrical capsule (Video S1)

- Magnetic attraction after two layer separation (Video S2)

\section{REFERENCES}

(1) Seliktar, D. Designing Cell-Compatible Hydrogels for Biomedical Applications. Sci. 2012, 336, 1124.

(2) Aguado, B. A.; Grim, J. C.; Rosales, A. M.; Watson-Capps, J. J.; Anseth, K. S. Engineering Precision Biomaterials for Personalized Medicine. Sci. Transl. Med. 2018, 10, 8645.

(3) Jones, G. B.; Collins, D. S.; Harrison, M. W.; Thyagarajapuram, N. R.; Wright, J. M. Subcutaneous Drug Delivery: An Evolving Enterprise. Sci. Transl. Med. 2017, 9, 9166.

(4) Mitragotri, S.; Burke, P. A.; Langer, R. Overcoming the Challenges in Administering Biopharmaceuticals: Formulation and Delivery Strategies. Nat. Rev. Drug Discov. 2014, 13, 655.

(5) Neslihan Gursoy, R.; Benita, S. Self-Emulsifying Drug Delivery Systems (Sedds) for Improved Oral Delivery of Lipophilic Drugs. Biomed. Pharmacother. 2004, 58, 173-182. 
(6) Dressman, J. B.; Amidon, G. L.; Reppas, C.; Shah, V. P. Dissolution Testing as a Prognostic Tool for Oral Drug Absorption: Immediate Release Dosage Forms. Pharm. Res-Dordr. 1998, 15, 11-22.

(7) Xiao, H.; Brazel, C. On the Importance and Mechanisms of Burst Release in Matrix-Controlled Drug Delivery Systems. J. Control. Release 2001, 73, 121-136.

(8) Liang, K.; Carmone, S.; Brambilla, D.; Leroux, J. C. 3d Printing of a Wearable Personalized Oral Delivery Device: A First-in-Human Study. Sci. Adv. 2018, 4, 2544.

(9) Goyanes, A.; Wang, J.; Buanz, A.; Martínez-Pacheco, R.; Telford, R.; Gaisford, S.; Basit, A. W. 3d Printing of Medicines: Engineering Novel Oral Devices with Unique Design and Drug Release Characteristics. Mol. Pharm. 2015, 12, 4077-4084.

(10) Khaled, S. A.; Burley, J. C.; Alexander, M. R.; Yang, J.; Roberts, C. J. 3d Printing of Five-in-One Dose Combination Polypill with Defined Immediate and Sustained Release Profiles. J. Control Release 2015, 217, 308-14.

(11) Wang, B. L.; Ahmad, Z.; Huang, J.; Li, J. S.; Chang, M. W. Development of Random and Ordered Composite Fiber Hybrid Technologies for Controlled Release Functions. Chem. Eng. J. 2018, 343, 379389.

(12) Gao, J.; Chen, C.; Chen, J. X.; Wen, L. M.; Yang, G. L.; Duan, F. P.; Huang, Z. Y.; Li, D. F.; Yu, D. R.; Yang, H. J.; Li, S. J. Synergism and Rules of the New Combination Drug Yiqijiedu Formulae (Yqjd) on Ischemic Stroke Based on Amino Acids (Aas) Metabolism. Sci. Rep. 2014, 4, 5149.

(13) Sherry, K. M.; Li, W.; Dulin, W.; Donahue, F.; Cade, D.; Benameur, H.; Hutchison, K. Performance Qualification of a New Hypromellose Capsule: Part I. Comparative Evaluation of Physical, Mechanical and Processability Quality Attributes of Vcaps Plus, Quali-V and Gelatin Capsules. Int.J. Pharm. 2010, 386, 30-41.

(14) Ciper, M.; Bodmeier, R. Modified Conventional Hard Gelatin Capsules as Fast Disintegrating Dosage Form in the Oral Cavity. Eur. J. Pharm. Biopharm. 2006, 62, 178-184.

(15) Cole, E. T.; Dominique, C.; Hassan, B. Challenges and Opportunities in the Encapsulation of Liquid and Semi-Solid Formulations into Capsules for Oral Administration. Adv. Drug Delivery Rev. 2008, 60, 747-756.

(16) Singh, M. N.; Hemant, K. S.; Ram, M.; Shivakumar, H. G. Microencapsulation: A Promising Technique for Controlled Drug Delivery. Res. Pharm. Sci. 2010, 5, 65-77.

(17) Po, A. L. W.; Mroso, P. V. Drug-Drug Incompatibility in the Solid State: Kinetic Interpretation, Modelling and Prediction. Int. J. Pharm. 1984, 18, 287-298.

(18) Robert, A.; Nezamis, J. E.; Lancaster, C.; Hanchar, A. J. Cytoprotection by Prostaglandins in Rats. Gastroenterology 1979, 77, 433-443.

(19) Schoen, R. T.; Vender, R. J. Mechanisms of Nonsteroidal Anti-Inflammatory Drug-Induced Gastric Damage. Am.J. Med. 1989, 86, 449-58.

(20) Wijnberg, I. D.; Ververs, F. F. Phenytoin Sodium as a Treatment for Ventricular Dysrhythmia in Horses. J. Vet. Intern. Med. 2010, 18, 350-353.

(21) Highley, C. B.; Rodell, C. B.; Burdick, J. A. Direct 3d Printing of Shear-Thinning Hydrogels into Self-Healing Hydrogels. Adv. Mater. 2015, 27, 5075-5079.

(22) Inzana, J. A.; Olvera, D.; Fuller, S. M.; Kelly, J. P.; Graeve, O. A.; Schwarz, E. M.; Kates, S. L.; Awad, H. A. 3d Printing of Composite Calcium Phosphate and Collagen Scaffolds for Bone Regeneration. Biomaterials 2014, 35, 4026-34.

(23) Ventola, C. L. Medical Applications for 3d Printing: Current and Projected Uses. P.\&T. 2014, 39, 704-711. 
(24) Kolesky, D. B.; Truby, R. L.; Gladman, A. S.; Busbee, T. A.; Homan, K. A.; Lewis, J. A. 3d Bioprinting of Vascularized, Heterogeneous Cell-Laden Tissue Constructs. Adv. Mater. 2014, 26, 31243130 .

(25) Goyanes, A.; Wang, J.; Buanz, A.; Martinez-Pacheco, R.; Telford, R.; Gaisford, S.; Basit, A. W. 3d Printing of Medicines: Engineering Novel Oral Devices with Unique Design and Drug Release Characteristics. Mol. Pharm. 2015, 12, 4077-84.

(26) Okwuosa, T. C.; Stefaniak, D.; Arafat, B.; Isreb, A.; Wan, K. W.; Alhnan, M. A. A Lower Temperature Fdm 3d Printing for the Manufacture of Patient-Specific Immediate Release Tablets. Pharm. Res. 2016, 33, 2704-12.

(27) Wang, J. C.; Chang, M. W.; Ahmad, Z.; Li, J. S. Fabrication of Patterned Polymer-Antibiotic Composite Fibers Via Electrohydrodynamic (Ehd) Printing. J. Drug Deliv. Sci. Tec. 2016, 35, 114-123.

(28) Wu, S. T.; Li, J. S.; Mai, J.; Chang, M. W. Three-Dimensional Electrohydrodynamic Printing and Spinning of Flexible Composite Structures for Oral Multidrug Forms. ACS Appl. Mater. Interfaces 2018, 10, 24876-24885.

(29) Yao, Z. C.; Wang, J. C.; Ahmad, Z.; Li, J. S.; Chang, M. W. Fabrication of Patterned ThreeDimensional Micron Scaled Core-Sheath Architectures for Drug Patches.Mater. Sci.Eng.C-Mater.Biol. Appl. 2019, 97, 776-783.

(30) Jang, Y.; Tambunan, I. H.; Tak, H.; Nguyen, V. D.; Kang, T.; Byun, D. Non-Contact Printing of High Aspect Ratio Ag Electrodes for Polycrystalline Silicone Solar Cell with Electrohydrodynamic Jet Printing. Appl. Phys. Lett. 2013, 102, 4.

(31) Wang, B.; Chen, X.; Ahmad, Z.; Huang, J.; Chang, M.-W. 3d Electrohydrodynamic Printing of Highly Aligned Dual-Core Graphene Composite Matrices. Carbon 2019, 153, 285-297.

(32) Yao, Z. C.; Wang, J. C.; Wang, B. L.; Ahmad, Z.; Li, J. S.; Chang, M. W. A Novel Approach for Tailored Medicines: Direct Writing of Janus Fibers. J. Drug Deliv. Sci. Tec. 2019, 50, 372-379.

(33) Bhardwaj, N.; Kundu. Electrospinning: A Fascinating Fiber Fabrication Technique. Biotechnol. Adv. 2010, 28, 325-347.

(34) Yao, Z. C.; Chang, M. W.; Ahmad, Z.; Li, J. S. Encapsulation of Rose Hip Seed Oil into Fibrous Zein Films for Ambient and on Demand Food Preservation Via Coaxial Electrospinning. J. Food Eng. 2016, 191, 115-123.

(35) Moriyama, K.; Yasuhara, Y.; Ota, H. Visualization of Protonation/Deprotonation of Active Pharmaceutical Ingredient in Solid State by Vapor Phase Amine-Selective Alkyne Tagging and Raman Imaging. J. Pharm. Sci. 2017, 106, 1778-1785.

(36) Medina, M. A.; Ramirez, F. J.; Ruiz-Chica, J.; Chavarria, T.; Lopez-Navarrete, J. T.; SanchezJimenez, F. DNA-Chlorpheniramine Interaction Studied by Spectroscopic Techniques. Biochim Biophys Acta 1998, 1379, 129-33.

(37) Al-Zoubi, N.; Koundourellis, J. E.; Malamataris, S. Ft-Ir and Raman Spectroscopic Methods for Identification and Quantitation of Orthorhombic and Monoclinic Paracetamol in Powder Mixes. $J$. Pharmaceut. Biomed. 2002, 29, 459-467.

(38) Ragelle, H.; Tibbitt, M. W.; Wu, S. Y.; Castillo, M. A.; Cheng, G. Z.; Gangadharan, S. P.; Anderson, D. G.; Cima, M. J.; Langer, R. Surface Tension-Assisted Additive Manufacturing. Nat. Commun. 2018, 9, 1184.

(39) Wu, Y.; Clark, R. L. Controllable Porous Polymer Particles Generated by Electrospraying. J. Colloid Interface Sci. 2007, 310, 529-35.

(40) Breitenbach, J.; Schrof, W.; Neumann, J. Confocal Raman-Spectroscopy: Analytical Approach to 
Solid Dispersions and Mapping of Drugs. Pharm. Res. 1999, 16, 1109-13.

(41) Trenfield, S. J.; Goyanes, A.; Telford, R. 3d Printed Drug Products Non-Destructive Dose Verification Using a Rapid Point-and-Shoot Approach. Int. J. Pharm. 2012, 9, 1861.

(42) Medina, M. A.; Ramirez, F. J.; Ruiz-Chica, J.; Chavarria, T.; Lopez-Navarrete, J. T.; SanchezJimenez, F. DNA-Chlorpheniramine Interaction Studied by Spectroscopic Techniques. Biochim. Biophys. Acta. 1998, 1379, 129-33.

(43) Wang, J.; Cheung, M. K.; Mi, Y. L. Miscibility and Morphology in Crystalline/Amorphous Blends of Poly(Caprolactone)/Poly(4-Vinylphenol) as Studied by Dsc, Ftir, and C-13 Solid State Nmr. Polymer 2002, 43, 1357-1364.

(44) Wang, H.; Qiao, X.; Chen, J.; Wang, X.; Ding, S. Mechanisms of Pvp in the Preparation of Silver Nanoparticles. Mater. Chem. Phys. 2005, 94, 449-453.

(45) Fujiwara, M.; Chow, P. S.; Ma, D. L.; Braatz, R. D. Paracetamol Crystallization Using Laser Backscattering and Atr-Ftir Spectroscopy: Metastability, Agglomeration, and Control. Cryst. Growth Des. 2002, 2, 363-370.

(46) Rajabalaya, R. Studies on Effect of Plasticizer on Invitro Release and Exvivo Permeation from Eouragit E100 Based Chlorpheniramine Maleate Maleate Matrix Type Transdermal Delivery System. $J$. Excip. Food Chem. 2010, 1, 3-12.

(47) Repka, M. A.; McGinity, J. W. Influence of Chlorpheniramine Maleate on Topical Hydroxypropylcellulose Films Produced by Hot-Melt Extrusion. Pharm. Dev. Technol. 2001, 6, 297-304. (48) Bustamante, P.; Romero, S.; Pena, A.; Escalera, B.; Reillo, A. Enthalpy-Entropy Compensation for the Solubility of Drugs in Solvent Mixtures: Paracetamol, Acetanilide, and Nalidixic Acid in DioxaneWater. J. Pharm. Sci. 1998, 87, 1590-1596.

(49) Meng, Z. X.; Zheng, W.; Li, L. Fabrication and Characterization of Three-Dimensional Nanofiber Membrance of Pcl-Mwcnts by Electrospinning. Mat. Sci. Eng. C 2010, 30, 1014-1021.

(50) Gautam, S.; Dinda, A. K.; Mishra, N. C. Fabrication and Characterization of Pcl/Gelatin Composite Nanofibrous Scaffold for Tissue Engineering Applications by Electrospinning Method. Mater. Sci. Eng. C Mater. Biol. Appl. 2013, 33, 1228-35.

(51) Razzak, M. T.; Zainuddin; Erizal; Dewi, S. P.; Lely, H.; Taty, E.; Sukirno. The Characterization of Dressing Component Materials and Radiation Formation of Pva-Pvp Hydrogel. Radiat. Phys. Chem. 1999, 55, 153-165.

(52) Wu, C.; McGinity, J. W. Non-Traditional Plasticization of Polymeric Films. Int. J. Pharm. 1999, $177,15-27$.

(53) Jenquin, M. R.; Mcginity, J. W. Characterization of Acrylic Resin Matrix Films and Mechanisms of Drug-Polymer Interactions. Int. J. Pharm. 1994, 101, 23-34.

(54) Wang, I. C.; Lee, M. J.; Seo, D. Y.; Lee, H. E.; Choi, Y.; Kim, W. S.; Kim, C. S.; Jeong, M. Y.; Choi, G. J. Polymorph Transformation in Paracetamol Monitored by in-Line Nir Spectroscopy During a Cooling Crystallization Process. AAPS PharmSciTech 2011, 12, 764-70.

(55) Qian, K. K.; Bogner, R. H. Application of Mesoporous Silicon Dioxide and Silicate in Oral Amorphous Drug Delivery Systems. J. Pharm. Sci. 2012, 101, 444-463.

(56) Laitinen, R.; Lobmann, K.; Strachan, C. J.; Grohganz, H.; Rades, T. Emerging Trends in the Stabilization of Amorphous Drugs. Int. J. Pharm. 2013, 453, 65-79.

(57) Sun, C.; Lee, J. S. H.; Zhang, M. Magnetic Nanoparticles in Mr Imaging and Drug Delivery. Adv. Drug Delivery Rev. 2008, 60, 1252-1265.

(58) Veiseh, O.; Gunn, J. W.; Zhang, M. Design and Fabrication of Magnetic Nanoparticles for Targeted 
Drug Delivery and Imaging. Adv. Drug Delivery Rev. 2010, 62, 284-304.

(59) María, V.; Francisco, B.; Daniel, A. Mesoporous Materials for Drug Delivery. Angew. Chem. Int. Edit. 2010, 46, 7548-7558.

(60) Huang, X.; Brazel, C. S. On the Importance and Mechanisms of Burst Release in Matrix-Controlled Drug Delivery Systems. J. Control. Release 2001, 73, 121-136.

(61) Bravo, S. A.; Lamas, M. C.; Salamon, C. J. In-Vitro Studies of Diclofenac Sodium ControlledRelease from Biopolymeric Hydrophilic Matrices. J. Pharm. Sci. 2002, 5, 213-9.

(62) S Sadish, K.; Kumar, Y.; Khan, M. S. Y.; Anbu, J.; Clercq, E.De. Antihistaminic and Antiviral Activities of Steroids of Turbinaria Conoides. Nat. Prod. Res. 2011, 25, 723-729.

\section{TOC Figure}
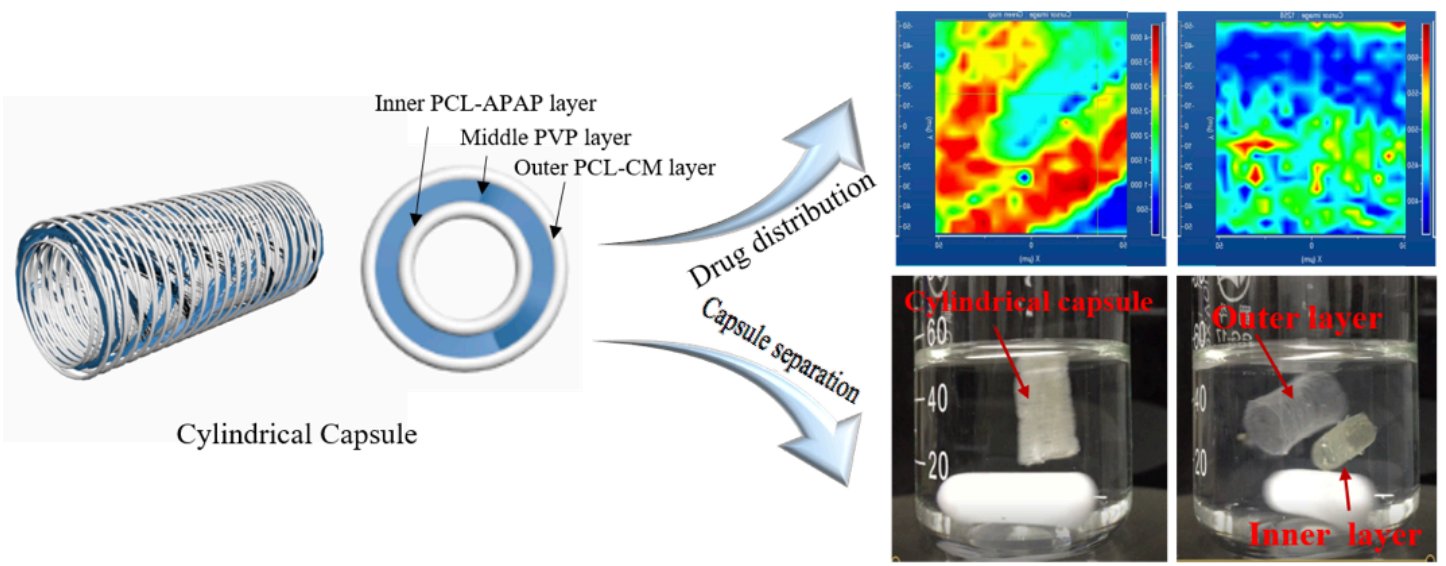